\title{
POISSON SAMPLING FOR SPECTRAL ESTIMATION IN PERIODICALLY CORRELATED PROCESSES
}

Abstract. We study estimation problems for periodically correlated, non gaussian processes. We estimate the correlation functions and the spectral densities from continuous-time samples. From a random time sample, we construct three types of estimators for the spectral densities and we prove their consistency.

1. Introduction. The processes we encounter in applications are often assumed to be stationary in the wide sense. Many problems pertaining to these processes have been studied. However, in practice, the stationary hypothesis is not always valid. In that case, we say that the processes are nonstationary. In this paper, we consider processes satisfying for all $s, t$,

$$
E(X(t+T))=E(X(t))
$$

and

$$
E(X(s+T) X(t+T))=E(X(s) X(t))
$$

where $T$ is a fixed nonnegative number, for example the noise of a periodic oscillator.

Such processes are called periodically correlated up to order two. They are encountered in meteorology, in communications and also in radio-physics. H. L. Hurd [4] has found estimators for the characteristics of periodically correlated processes from continuous-time samples. He showed these estimators are consistent if the process is gaussian. We will show the consistency of these estimators under certain conditions on the fourth cumulant function (the process is not assumed to be gaussian). However, numerical computation of the estimators leads to a discretization of the process. Thus, it is better to estimate the characteristics from discrete samples (periodic or

1991 Mathematics Subject Classification: 62M10, 62M99, 62G07.

Key words and phrases: periodically correlated processes, Poisson sampling, quarticmean consistency, spectral density functions. 
random). We will see that these results are valid for almost periodically correlated harmonizable gaussian processes.

\section{Generalities}

Definition. A second order real process $X(t)$ is called periodically correlated up to order 2 with period $T$ if

$$
\forall t \in \mathbb{R}, \quad E\{X(t)\}=m(t)=m(t+T)
$$

and

$$
\forall s, t \in \mathbb{R}, \quad E\{X(s) X(t)\}=R(s, t)=R(s+T, t+T) .
$$

If $X(t)$ is periodically correlated, then

$$
B(t, u)=R(t+u, t),
$$

the autocovariance function of the process, is periodic in $t$ with period $T$. For fixed $u$, we can suppose that $B(\cdot, u) \in L^{1}[0, T]$ and so we assume the Fourier series representation

$$
B(t, u)=\sum_{k=-\infty}^{\infty} B_{k}(u) \exp \left(i \frac{2 \pi}{T} k t\right)
$$

where the coefficient functions are given by

$$
B_{k}(u)=\frac{1}{T} \int_{0}^{T} B(t, u) \exp \left(-i \frac{2 \pi}{T} k t\right) d t \quad(k \in \mathbb{Z}) .
$$

If we assume that $B_{k}(\cdot) \in L^{1}(\mathbb{R})$, then

$$
g_{k}(\omega)=\frac{1}{2 \pi} \int_{-\infty}^{\infty} B_{k}(u) \exp (-i \omega u) d u
$$

exists for $k \in \mathbb{Z}$. The functions $g_{k}(\omega)(k \in \mathbb{Z})$ are called the spectral density functions of $X(t)$. For $B_{k}(\cdot)$ and $g_{k}(\cdot)$, we have the following properties:

$$
\begin{aligned}
& B_{k}(-u)=\exp \left(-i \frac{2 \pi}{T} k u\right) B_{k}(u) \\
& g_{k}(\omega)=\frac{1}{2 \pi} \int_{0}^{\infty}\left[\exp (-i \omega u)+\exp \left(i\left(\omega-\frac{2 \pi}{T} k\right)\right)\right] B_{k}(u) d u, \\
& g_{k}(\omega)=g_{k}\left(\frac{2 \pi}{T} k-\omega\right) \\
& \overline{g_{k}(\omega)}=g_{-k}(-\omega) .
\end{aligned}
$$

Definition. A real process $X(t)$ having finite moments of order $p$ is called periodically correlated up to order $p$ with period $T$ if for $t_{1}, \ldots, t_{n} \in \mathbb{R}$ 
and for $p_{1}, \ldots, p_{n} \in \mathbb{N}$ satisfying $\sum_{i=1}^{n} p_{i} \leq p$,

$$
E\left\{X\left(t_{1}+T\right)^{p_{1}} \ldots X\left(t_{n}+T\right)^{p_{n}}\right\}=E\left\{X\left(t_{1}\right)^{p_{1}} \ldots X\left(t_{n}\right)^{p_{n}}\right\} .
$$

We notice that if $X(t)$ is $\mathrm{PC}$ up to order $p$ then $X(t)$ is $\mathrm{PC}$ up to order $q \leq p$. Throughout the paper, the term $P C$ processes will be used for all periodically correlated processes up to order 2 .

Assuming $X(t)$ is $\mathrm{PC}$ up to order 4 , the functions

$$
\nu\left(t, u_{1}, u_{2}, u_{3}\right)=E\left(X(t) X\left(t+u_{1}\right) X\left(t+u_{2}\right) X\left(t+u_{3}\right)\right)
$$

and

$$
\begin{aligned}
K\left(t, u_{1}, u_{2}, u_{3}\right)= & \nu\left(t, u_{1}, u_{2}, u_{3}\right)-B\left(t, u_{1}\right) B\left(t+u_{2}, u_{3}-u_{2}\right) \\
& -B\left(t, u_{2}\right) B\left(t+u_{3}, u_{1}-u_{2}\right) \\
& -B\left(t, u_{3}\right) B\left(t+u_{1}, u_{2}-u_{1}\right)
\end{aligned}
$$

are periodic in $t$ with period $T$. The last function is called the fourth cumulant function. We see that if $X(t)$ is gaussian, then $K$ is identically zero.

3. Estimation from continuous-time samples. We assume throughout that

(1) $X(t)$ is PC up to order 4 ,

(2) $X(t)$ has uniformly bounded fourth moment, $E\left\{X(t)^{4}\right\} \leq M$,

(3) $m(t) \equiv 0$.

From a sample of $X(t), 0 \leq t \leq A$, let us estimate:

- $B_{k}(u)$ by

$$
\widehat{B}_{k}(A, u)= \begin{cases}\frac{1}{A} \int_{0}^{A-u} X(t) X(t+u) \exp \left(-i \frac{2 \pi}{T} k t\right) d t \quad \text { if } u \geq 0, \\ \frac{1}{A} \int_{-u}^{A} X(t) X(t+u) \exp \left(-i \frac{2 \pi}{T} k t\right) d t \quad \text { if } u<0,\end{cases}
$$

- $g_{k}(\omega)$ by

$$
\begin{aligned}
\widehat{g}_{k}(A, \omega) & =\frac{1}{2 \pi} \int_{-A}^{A} h\left(B_{A} v\right) \widehat{B}_{k}(A, v) \exp (-i \lambda v) d v \\
& =\frac{1}{B_{A}} \int_{-\infty}^{\infty} H\left(\frac{u-\lambda}{B_{A}}\right) g_{k}(A, u) d u
\end{aligned}
$$

where

$$
g_{k}(A, \lambda)=\frac{1}{2 \pi A} I_{A}(\lambda) \overline{I_{A}\left(\lambda-\frac{2 \pi}{T} k\right)}
$$


with $I_{A}(\lambda)=\int_{0}^{A} X(t) \exp (-i \lambda t) d t ; B_{A}$ is a nonnegative function of $A$ for which $\lim _{A \rightarrow \infty} B_{A}=0 ; h$ is an even, integrable function for which $|h(t)|$ $\leq M^{\prime}$ for $t, h(0)=1$, and $H(\omega)=\int_{-\infty}^{\infty} h(t) \exp (-i \omega t) d t$.

Proposition 3.1. If $X(t)$ is PC up to order 4 with $\int_{-\infty}^{\infty} \int_{0}^{T} B(t, u)^{2} d t d u$ $<\infty$ and

$$
\forall u \in \mathbb{R}, \quad \int_{-\infty}^{\infty} \int_{0}^{T}|K(t, u, v, v+u)| d t d v<\infty
$$

then

$$
\forall u \in \mathbb{R}, \quad \lim _{A \rightarrow \infty} E\left\{\left|\widehat{B}_{k}(A, u)-B_{k}(u)\right|^{2}\right\}=0 .
$$

Proof. The proof will be given for fixed $u \geq 0$. Let

$$
\begin{aligned}
J_{k}(A) & =\frac{1}{A} \int_{0}^{A-u}(X(t+u) X(t)-B(t, u)) \exp \left(-i \frac{2 \pi}{T} k t\right) d t \\
& =\widehat{B}_{k}(A, u)-E\left\{\widehat{B}_{k}(A, u)\right\} .
\end{aligned}
$$

It suffices to show that $\lim _{A \rightarrow \infty} E\left\{\left|J_{k}(A)\right|^{2}\right\}=0$ since

$$
E\left\{\left|\widehat{B}_{k}(A, u)-B_{k}(u)\right|^{2}\right\}^{1 / 2} \leq E\left\{\left|J_{k}(A)\right|^{2}\right\}^{1 / 2}+\left|E\left(\widehat{B}_{k}(A, u)\right)-B_{k}(u)\right| .
$$

Using (2.2), we have

$$
\begin{aligned}
E\left\{\left|J_{k}(A)\right|^{2}\right\} \leq & \frac{1}{A^{2}} \int_{0}^{A-u} \int_{0}^{A-u}|B(s-t, t+u) B(s-t, t)| d s d t \\
& +\frac{1}{A^{2}} \int_{0}^{A-u} \int_{0}^{A-u}|B(t, s-t+u) B(s, t-s+u)| d s d t \\
& +\frac{1}{A^{2}} \int_{0}^{A-u} \int_{0}^{A-u}|K(s, u, t-s, t-s+u)| d s d t .
\end{aligned}
$$

The first and second terms go to zero as $A$ goes to infinity ([4], Prop. 6). Let us consider the third expression:

$$
\begin{aligned}
& \frac{1}{A^{2}} \int_{0}^{A-u} \int_{0}^{A-u}|K(s, u, t-s, t-s+u)| d s d t \\
& \quad \leq \frac{1}{A^{2}} \int_{-\infty}^{\infty} \int_{0}^{2 A}|K(s, u, t, t+u)| d s d t \\
& \quad \leq \frac{1}{A^{2}}\left(1+\left[\frac{2 A}{T}\right]\right) \int_{-\infty}^{\infty} d t \int_{0}^{T}|K(s, u, t, t+u)| d s=O\left(\frac{1}{A}\right)
\end{aligned}
$$

where $[x]$ is the integer part of $x$. 
Proposition 3.2. Let $X(t)$ be PC up to order 4 , with $K \in L^{1}\left([0, T] \times \mathbb{R}^{3}\right)$ and

$$
\int_{-\infty}^{\infty}\left(\int_{0}^{T} B(t, u)^{2} d t\right)^{1 / 2} d u<\infty
$$

Then, for all $\omega_{1}, \omega_{2} \geq 0$ and for all $j, k \in \mathbb{Z}$,

$$
\begin{aligned}
\lim _{A \rightarrow \infty} A B_{A} \mid \operatorname{cov} & \left\{\widehat{g}_{j}\left(A, \omega_{1}\right), \widehat{g}_{k}\left(A, \omega_{2}\right)\right\} \mid \\
\leq & \frac{2}{2 \pi^{2}} \int_{0}^{\infty}|h(z)|^{2} d z \int_{-\infty}^{\infty} d u_{1} \int_{-\infty}^{\infty} S\left(u+u_{1}\right) S(u) d u<\infty
\end{aligned}
$$

where $S(u)^{2}=T^{-1} \int_{0}^{T} B(t, u)^{2} d t=\sum_{k=-\infty}^{\infty}\left|B_{k}(u)\right|^{2}$.

Proof. We have

$$
\begin{aligned}
A B_{A} \operatorname{cov}\left\{\widehat{g}_{j}\left(A, \omega_{1}\right), g_{k}\left(A, \omega_{2}\right)\right\} \\
=\frac{A B_{A}}{2 \pi^{2}} \int_{-A}^{A} \int_{-A}^{A} h\left(B_{A} v_{1}\right) h\left(B_{A} v_{2}\right) \\
\quad \times \operatorname{cov}\left\{\widehat{B}_{j}\left(A, v_{1}\right), \widehat{B}_{k}\left(A, v_{2}\right)\right\} \exp \left(-i \omega_{1} v_{1}+i \omega_{2} v_{2}\right) d v_{1} d v_{2} \\
=I_{1}+I_{2}+I_{3}
\end{aligned}
$$

where

$$
\begin{aligned}
& I_{1}=\frac{1}{A^{2}} \int_{0}^{A-v_{1}} \int_{0}^{A-v_{2}} B\left(t+v_{2}, s+v_{1}-t\right.\left.-v_{2}\right) B(t, s-t) \\
& \times \exp \left(-i \frac{2 \pi}{T}(j s-k t)\right) d s d t \\
& I_{2}=\frac{1}{A^{2}} \int_{0}^{A-v_{1}} \int_{0}^{A-v_{2}} B\left(t, s-t+v_{2}\right) B\left(t+v_{2}, s-t-v_{2}\right) \\
& \times \exp \left(-i \frac{2 \pi}{T}(j s-k t)\right) d s d t \\
& I_{3}=\frac{1}{A^{2}} \int_{0}^{A-v_{1}} \int_{0}^{A-v_{2}} K\left(t, v_{2}, s-t, s-t+v_{1}\right) \exp \left(-i \frac{2 \pi}{T}(j s-k t)\right) d s d t
\end{aligned}
$$

and

$$
\lim _{A \rightarrow \infty}\left(\left|I_{1}\right|+\left|I_{2}\right|\right) \leq \frac{C}{2 \pi^{2}} \int_{0}^{\infty}|h(z)|^{2} d z \int_{-\infty}^{\infty} d u_{1} \int_{-\infty}^{\infty} S\left(u+u_{1}\right) S(u) d u
$$

([4], Th. 3 and Prop. 7). 
On the other hand,

$$
\begin{aligned}
\left|I_{3}\right| \leq & \frac{4 B_{A}}{2 \pi^{2} A} \int_{-A}^{A} \int_{-A}^{A}\left|h\left(B_{A} v_{1}\right) h\left(B_{A} v_{2}\right)\right| \\
& \times\left(\int_{0}^{2 A} \int_{0}^{2 A}\left|K\left(t, v_{2}, s-t, s-t+v_{1}\right)\right| d s d t\right) d v_{1} d v_{2} \\
\leq & \frac{2 M^{2} B_{A}}{\pi^{2} A}\left(1+\left[\frac{2 A}{T}\right]\right) \int_{0}^{T} \int_{\mathbb{R}^{3}}\left|K\left(t, u_{1}, u_{2}, u_{3}\right)\right| d t d u_{1} d u_{2} d u_{3} \\
= & O\left(B_{A}\right) .
\end{aligned}
$$

If we choose $B_{A}$ so that $\lim _{A \rightarrow \infty} A B_{A}=\infty$, we obtain the consistency of the estimator $\widehat{g}_{k}(A, \omega)$.

4. Estimation from periodic sampling. Let $X(t)$ be a PC process with period $T$ and with autocovariance function

$$
B(t, u)=\sum_{k \in \mathbb{Z}} B_{k}(u) \exp \left(i \frac{2 \pi}{T} k t\right) .
$$

Let $h>0$; consider the process $Y_{n}=X(n h)$ with $n \in \mathbb{Z}$. By analogy with the stationary case, we can look if it is possible to estimate the characteristics of $X(t)$ with a sample from $Y_{n}$. Let $B^{h}(m, n)=E\left(Y_{m+n} Y_{m}\right)$, which is the autocovariance function of $Y_{n}$. Assume first that $T / h=N$ is a nonnegative integer; then for all integers $m$ and $n, B^{h}(m+N, n)=B^{h}(m, n)$, therefore $Y_{n}$ is $\mathrm{PC}$ in discrete time. In this case, we can write

with

$$
B^{h}(m, n)=\sum_{l=0}^{N-1} B_{l}^{h}(n) \exp \left(i \frac{2 \pi}{N} m l\right)
$$

On the other hand,

$$
B_{l}^{h}(n)=\frac{1}{N} \sum_{m=0}^{N-1} B^{h}(m, n) \exp \left(-i \frac{2 \pi}{N} m l\right) .
$$

$$
\begin{aligned}
B^{h}(m, n) & =B(m h, n h)=\sum_{k=-\infty}^{\infty} B_{k}(n h) \exp \left(i \frac{2 \pi}{T} k m h\right) \\
& =\sum_{l=0}^{N-1} \sum_{k=-\infty}^{\infty} B_{j N+l}(n h) \exp \left(i \frac{2 \pi}{N}(j N+l) m\right) \\
& =\sum_{l=0}^{N-1}\left(\sum_{j=-\infty}^{\infty} B_{j N+l}(n h)\right) \exp \left(i \frac{2 \pi}{N} l m\right),
\end{aligned}
$$

so $B_{l}^{h}(n)=\sum_{j=-\infty}^{\infty} B_{j N+l}(n h)$. 
We will show that periodic sampling involves aliasing, that is, we can find two different autocovariance functions $B_{1}(t, u), B_{2}(t, u)$ such that $B_{1}^{h}(m, n)$ $=B_{2}^{h}(m, n)$ for all $m$ and $n$. In this case, we say that $B_{1}(t, u)$ and $B_{2}(t, u)$ are aliases. To show that, for fixed $u$, assume $B(t, u) \in L^{2}[0, T]$ and let for $k \in \mathbb{Z}$

$$
B_{p, k}(u)=\cos \left(\frac{2 \pi}{T} p u\right) B_{k}(u)
$$

where $p$ is a nonzero integer. For fixed $p, B_{p, k}$ and $B_{k}$ are two different functions of $u$, so, in $L^{2}[0, T]$, the function

$$
B_{p}(t, u)=\sum_{k \in \mathbb{Z}} B_{p, k}(u) \exp \left(i \frac{2 \pi}{T} k t\right)
$$

is different from $B(t, u)$.

$B_{p}(t, u)$ is the autocovariance function of a PC process with period $T$. In fact ([3], Theorem 1), it suffices to show that for integers $k_{1}, \ldots, k_{n}$, real numbers $u_{1}, \ldots, u_{n}$, and complex numbers $x_{1}, \ldots, x_{n}$,

$$
A=\sum_{p, q=1}^{n} x_{p} \bar{x}_{q} B_{p, k_{p} k_{q}}\left(u_{p}-u_{q}\right) \geq 0
$$

with

$$
\begin{aligned}
B_{p, j k}(u) & =B_{p, k-j}(u) \exp \left(i \frac{2 \pi}{T} j u\right) \\
& =\frac{1}{2}\left\{\exp \left(i \frac{2 \pi}{h} p u\right)+\exp \left(-i \frac{2 \pi}{h} p u\right)\right\} B_{j k}(u)
\end{aligned}
$$

where $B_{j k}(u)=B_{k-j} \exp \left(i \frac{2 \pi}{T} j u\right)$. Now

$$
\begin{aligned}
2 A= & \sum_{i, j=1}^{n} x_{i} \bar{x}_{j} \exp \left\{i \frac{2 \pi}{h}\left(u_{i}-u_{j}\right)\right\} B_{k_{i} k_{j}}\left(u_{i}-u_{j}\right) \\
& +\sum_{i, j=1}^{n} x_{i} \bar{x}_{j} \exp \left\{-i \frac{2 \pi}{h}\left(u_{i}-u_{j}\right)\right\} B_{k_{i} k_{j}}\left(u_{i}-u_{j}\right) \\
= & \sum_{i, j=1}^{n} x_{i} \exp \left(i \frac{2 \pi}{h} u_{i}\right) \overline{x_{j} \exp \left(i \frac{2 \pi}{h} u_{j}\right)} B_{k_{i} k_{j}}\left(u_{i}-u_{j}\right) \\
& +\sum_{i, j=1}^{n} x_{i} \exp \left(-i \frac{2 \pi}{h} u_{i}\right) \overline{x_{j} \exp \left(-i \frac{2 \pi}{h} u_{j}\right)} B_{k_{i} k_{j}}\left(u_{i}-u_{j}\right) \geq 0,
\end{aligned}
$$

since $B(t, u)$ is the autocovariance function of a PC process with period $T$. 
On the other hand,

$$
\sum_{j=-\infty}^{\infty} B_{p, j N+l}(n h)=\sum_{j=-\infty}^{\infty} B_{j N+l}(n h) \cos \left(\frac{2 \pi}{h} p n h\right)=\sum_{j=-\infty}^{\infty} B_{j N+l}(n h)
$$

and therefore $B_{p}^{h}(m, n)=B^{h}(m, n)$.

When $T / h$ is not an integer, let $r(u)$ denote any real even function in $L^{1} \cap L^{2}$, having a continuous second derivative and vanishing at the points $t_{n}=n h$ for which $r^{\prime}(0)=r^{\prime \prime}(0)=0$. For example, we can take $r$ so that on ]$t_{n}, t_{n+1}[$,

$$
r(u)=\exp \left[\frac{1}{\left(u+t_{n}+h / 2\right)^{2}-h^{2} / 4}\right] .
$$

Define $a_{k}(u)$ by

$$
\begin{gathered}
a_{0}(u)=a_{0}(-u)=r(u) \quad \text { for } u \geq 0, \\
a_{k}(u)=\frac{1}{k^{2}} r(u) \quad \text { and } \quad a_{k}(-u)=\exp \left(-i \frac{2 \pi}{T} k u\right) a_{k}(u) \\
\text { for } k \geq 1 \text { and } u \geq 0, \\
a_{-k}(u)=\overline{a_{k}(u)} \quad \text { for } k \geq 1 \text { and } u \in \mathbb{R} .
\end{gathered}
$$

The functions $a_{k}(u)$ have continuous second derivatives since $r^{\prime}(0)=$ $r^{\prime \prime}(0)=0$, vanishing at the points $t_{n}=n h$. We also have $a_{k} \in L^{1} \cap L^{2}$.

For $j, k \in \mathbb{Z}$, let

$$
\begin{aligned}
& a_{j k}(u)=a_{k-j}(u) \exp \left(i \frac{2 \pi}{T} j u\right), \\
& A_{j k}(\omega)=\frac{1}{2 \pi} \int_{-\infty}^{\infty} a_{j k}(\omega) \exp (-i \omega u) d u .
\end{aligned}
$$

We have

$$
\begin{gathered}
a_{j k}(-u)=\overline{a_{j k}(u)}, \quad a_{p-j, q-j}(u) \exp \left(i \frac{2 \pi}{T} j u\right)=a_{p q}(u), \\
A_{j k}(\omega)=\overline{A_{k j}(\omega)}, \quad A_{p-j, q-j}\left(\omega-\frac{2 \pi}{T} j\right)=A_{p q}(\omega) \quad \text { and } \quad A_{j k} \in L^{1} \cap L^{2} .
\end{gathered}
$$

For $\omega \in\left[0,2 \pi / T\left[\right.\right.$, there exists $A_{j k}^{(i)}, i=1,2$, so that $\left[A_{j k}^{(i)}(\omega)\right]_{j, k}, i=$ 1,2 , are nonnegative hermitian matrices, $\sup _{i=1,2}\left\{\left|A_{j k}^{(i)}(\omega)\right|\right\} \leq A_{j k}(\omega)$ and $A_{j k}(\omega)=A_{j k}^{(1)}(\omega)-A_{j k}^{(2)}(\omega)$. Thus for $\omega \in\left[\frac{2 \pi}{T} j, \frac{2 \pi}{T}(j+1)[\right.$, let for $i=1,2$,

$$
A_{j k}^{(i)}(\omega)=A_{p-j, q-j}^{(i)}\left(\omega-\frac{2 \pi}{T} j\right), \quad p, q \in \mathbb{Z},
$$




$$
B_{j k}^{(i)}(u)=\int_{-\infty}^{\infty} A_{j k}^{(i)}(u) \exp (i \omega u) d \omega=B_{0, k-j}^{(i)}(u) \exp \left(i \frac{2 \pi}{T} j u\right)
$$

Therefore putting $B_{k}^{(i)}(u)=B_{0, k}^{(i)}(u)$, we have

$$
B_{j k}^{(i)}(u)=B_{k-j}^{(i)}(u) \exp \left(i \frac{2 \pi}{T} j u\right) .
$$

We can easily verify that $\left|B_{k}^{(i)}(u)\right| \leq 1 / k^{2}$ for $k \neq 0$, therefore

$$
B^{(i)}(t, u)=\sum_{k \in \mathbb{Z}} B_{k}^{(i)}(u) \exp \left(i \frac{2 \pi}{T} j u\right)
$$

is well defined. We have

$$
\begin{aligned}
B_{j k}^{(1)}\left(t_{n}\right)-B_{j k}^{(2)}\left(t_{n}\right) & =\int_{-\infty}^{\infty}\left(A_{j k}^{(1)}(\omega)-A_{j k}^{(2)}(\omega)\right) \exp \left(i \omega t_{n}\right) d \omega \\
& =a_{j k}\left(t_{n}\right)=0,
\end{aligned}
$$

therefore $B_{k}^{(1)}\left(t_{n}\right)=B_{k}^{(2)}\left(t_{n}\right)$ and for all $m, n \in \mathbb{Z}, B^{(1)}\left(t, t_{n}\right)=B^{(2)}\left(t, t_{n}\right)$, $B^{(1)}\left(t_{m}, t_{n}\right)=B^{(2)}\left(t_{m}, t_{n}\right)$. We can easily show that for $i=1,2, B^{(i)}(t, u)$ is the autocovariance function of a PC process with period $T$. We conclude therefore that $B^{(1)}(t, u)$ and $B^{(2)}(t, u)$ are aliases. More generally, the family $B_{\alpha}(t, u)=\alpha B^{(1)}(t, u)+(1-\alpha) B^{(2)}(t, u)$, with $0 \leq \alpha \leq 1$, is a family of alias functions.

\section{Estimation from random samples}

(a) General case. Let $X(t)$ be a PC process with period $T$ for which the autocovariance function

$$
B(t, u)=\sum_{k \in \mathbb{Z}} B_{k}(u) \exp \left(-i \frac{2 \pi}{T} k t\right)
$$

and $B(t, u) \in L^{1}([0, T] \times \mathbb{R})$. We assume that the spectral density functions $g_{k}$ belong to $L^{1} \cap L^{2}$. Let $\left(t_{n}\right)_{n \geq 0}$ be a nondecreasing sequence of random variables such that $t_{0}=0$ and $t_{n}=\alpha_{n}+t_{n-1}$, where $\left(\alpha_{n}\right)_{n \geq 1}$ is a sequence of identically distributed, independent random variables, with $E \alpha_{n}<\infty$ and common probability density $f(u)$, independent of $X(t)$. We assume that $f \in L^{2}(\mathbb{R})$ and that $f(u)=0$ for $u \leq 0$, which implies that $t_{n} \geq t_{n-1}$. Let $f_{k}(u)$ be the probability density function of the sum of $k$ random variables $\alpha_{i}$. Then if $\phi(\omega)$ is the characteristic function of $\alpha_{i}$,

$$
\phi(\omega)^{k}=\int_{-\infty}^{\infty} f_{k}(u) \exp (i \omega u) d u
$$


is the characteristic function of the sum of the $\alpha_{i}$. Using Parseval's theorem, we get

$$
\begin{aligned}
\int_{-\infty}^{\infty} f_{k}(u)^{2} d u & =\int_{-\infty}^{\infty}|\phi(\omega)|^{2 k} d \omega \leq \frac{1}{2 \pi} \int_{-\infty}^{\infty}|\phi(\omega)|^{2} d \omega \\
& \leq \int_{-\infty}^{\infty} f(u)^{2} d u<\infty,
\end{aligned}
$$

therefore $f_{k} \in L^{2}$ and $\phi^{k} \in L^{2}$. Finally, we assume that for $u \geq 0$,

$$
\sum_{k \geq 1} f_{k}(u)=K<\infty \text {. }
$$

Let us now consider the process $Y_{n}=X\left(t_{n}\right), n \geq 0$; for $n \geq 0$, let

$$
C_{k}^{\alpha}(n)=\frac{1}{K T} \sum_{l \geq 1} E\left[Y_{l+n} Y_{l} \exp \left(-i \frac{2 \pi}{T} k t_{l}\right) 1_{\left\{t_{l}<T\right\}}\right] .
$$

For $n \geq 1$, we have

$$
\begin{aligned}
E\left[Y_{l+n} Y_{l}\right. & \left.\exp \left(-i \frac{2 \pi}{T} k t_{l}\right) 1_{\left\{t_{l}<T\right\}}\right] \\
& =E\left[E\left\{X\left(t_{l+n}\right) X\left(t_{l}\right) \exp \left(-i \frac{2 \pi}{T} k t_{l}\right) 1_{\left\{t_{l}<T\right\}} \mid\left(t_{n}\right)\right\}\right] \\
& =E\left[B\left(t_{l}, t_{l+n}-t_{l}\right) \exp \left(-i \frac{2 \pi}{T} k t_{l}\right) 1_{\left\{t_{l}<T\right\}}\right] \\
& =\int_{-\infty}^{\infty} \int_{0}^{T} B(t, u) \exp \left(-i \frac{2 \pi}{T} k t\right) f_{l}(t) f_{n}(u) d t d u,
\end{aligned}
$$

since $t_{l+n}-t_{l}$ with density $f_{n}$ and $t_{l}$ with density $f_{l}$ are independent. On the other hand,

$$
\begin{aligned}
& \sum_{l \geq 1} \int_{-\infty}^{\infty} \int_{0}^{T} \mid B(t, u) \exp \left(-i \frac{2 \pi}{T} k t\right) f_{l}(t) f_{n}(u) \mid d t d u \\
& \leq K \int_{-\infty}^{\infty} \int_{0}^{T}|B(t, u)| d t d u<\infty
\end{aligned}
$$

so, for $n \geq 1$, we have

$$
\begin{aligned}
C_{k}^{\alpha}(n) & =\frac{1}{K T} \sum_{l \geq 1} E\left[Y_{l+n} Y_{l} \exp \left(-i \frac{2 \pi}{T} k t_{l}\right) 1_{\left\{t_{l}<T\right\}}\right] \\
& =\int_{-\infty}^{\infty}\left\{\frac{1}{T} \int_{0}^{T} B(t, u) \exp \left(-i \frac{2 \pi}{T} k t\right) d t\right\} f_{n}(u) d u
\end{aligned}
$$




$$
=\int_{-\infty}^{\infty} B_{k}(u) f_{n}(u) d u=\int_{0}^{\infty} B_{k}(u) f_{n}(u) d u .
$$

Since

$B_{k}(u)=\int_{-\infty}^{\infty} g_{k}(\omega) \exp (i \omega u) d \omega$ and $f_{n}(u)=\frac{1}{2 \pi} \int_{-\infty}^{\infty} \phi(\omega)^{n} \exp (-i \omega u) d \omega$, we have, for $n \geq 1$,

$$
C_{k}^{\alpha}(n)=\int_{-\infty}^{\infty} g_{k}(\omega) \phi(\omega)^{n} d \omega
$$

On the other hand,

$$
\begin{aligned}
C_{k}^{\alpha}(0) & =\frac{1}{K T} \sum_{l \geq 1} E\left[X\left(t_{l}\right)^{2} \exp \left(-i \frac{2 \pi}{T} k t_{l}\right) 1_{\left\{t_{l}<T\right\}}\right] \\
& =\sum_{l \geq 1} \int_{0}^{T} B(t, 0) \exp \left(-i \frac{2 \pi}{T} k t\right) f_{l}(t) d t \\
& =B_{k}(0)=\int_{-\infty}^{\infty} g_{k}(\omega) d \omega .
\end{aligned}
$$

Finally, for $n \geq 0$ and $k \in \mathbb{Z}$, we have

$$
C_{k}^{\alpha}(n)=\int_{-\infty}^{\infty} g_{k}(\omega) \phi(\omega)^{n} d \omega .
$$

Proposition 5.1. Random sampling is alias-free if the characteristic function $\phi(\omega)$ takes no value more than once on the real axis.

Proof. For $k \in \mathbb{Z}, g_{k} \in L^{1} \cap L^{2}$, and it suffices to apply Theorem 1 of $[8]$ and (5.2).

Proposition 5.2. If the characteristic function $\phi(s)$ (where $s \in \mathbb{C}$, $\Im(s) \leq 0)$ takes the same value at two different points of the open upper half-plane, then aliasing occurs with random sampling.

Proof. We will show that for $k \geq 0$, there exist $A_{k} \in L^{1} \cap L^{2}$ not identically zero so that $A_{k}(\omega)=A_{k}\left(\frac{2 \pi}{T} k-\omega\right)$ and $\int_{-\infty}^{\infty} A_{k}(\omega) \phi(\omega)^{n} d \omega=0$ for $n \geq 0$. As Fourier transformation is a unitary transformation of $L^{2}$ onto itself, if $a_{k}(u)=\int_{-\infty}^{\infty} A_{k}(\omega) \exp (i \omega u) d \omega$ for $k \geq 0$ and $\mathcal{A}$ is the class of Fourier transforms of $L^{1}$ functions, we have to show that there exists a function $a_{k} \in \mathcal{A} \cap L^{2}$ not identically zero so that

$$
a_{k}(-u)=\exp \left(-i \frac{2 \pi}{T} k u\right) a_{k}(u),
$$




$$
\int_{0}^{\infty} a_{k}(u) f_{n}(u) d u=0 \quad(n \geq 1)
$$

$$
a_{k}(0)=\int_{-\infty}^{\infty} A_{k}(\omega) d \omega=0
$$

The existence of $a_{k}$ may be proved as in the proof of Theorem 2 of [8], p. 239 . Assume that $A_{k}$ is defined for $k \geq 0$. For $k<0$, let $A_{k}(-\omega)=\overline{A_{-k}(\omega)}$ and for $k, j \in \mathbb{Z}, A_{j k}(\omega)=A_{k-j}\left(\omega-\frac{2 \pi}{T} j\right)$. We have

$$
\begin{aligned}
\overline{A_{j k}(\omega)} & =\overline{A_{k-j}\left[\frac{2 \pi}{T}(k-j)-\left(\omega-\frac{2 \pi}{T} j\right)\right]} \\
& =A_{j-k}\left(\omega-\frac{2 \pi}{T} k\right)=A_{k j}(\omega) .
\end{aligned}
$$

We find $A_{j k}(\omega)$ which have the same properties as the properties used to construct alias autocovariance functions.

(b) Poisson sampling case. We assume throughout the paper that $\alpha_{n}$ are r.v. with common exponential density probability, $f(u)=\beta \exp (-\beta u) 1_{\mathbb{R}^{+}}(u)$; thus

$$
f_{n}(u)=\beta \exp (-\beta u) \frac{(\beta u)^{n-1}}{(n-1) !} 1_{\mathbb{R}^{+}}(u), \quad \phi(\omega)=\frac{\beta}{\beta-i \omega}
$$

and $\sum_{n \geq 1} f_{n}(u)=\beta<\infty$. It is easily seen from Proposition 5.1 that Poisson sampling is alias-free. The functions $\left\{f_{n}(u): n \geq 1\right\}$ form a complete system in $L^{2}\left[0, \infty\left[\right.\right.$, therefore we have a complete orthogonal sequence $\left\{q_{n}(u)\right.$ : $n \geq 1\}$ in $L^{2}\left[0, \infty\left[\right.\right.$ such that $q_{n}(u)=b_{n, 1} f_{1}(u)+\ldots+b_{n, n} f_{n}(u)$ where

$$
b_{n, l}=\left(\frac{2}{\beta}\right)^{1 / 2}(-2)^{l-1} \mathcal{C}_{n-1}^{l-1} .
$$

For $n \geq 1$, let

$$
\gamma_{k}^{\alpha}(n)=\int_{0}^{\infty} B_{k}(u) q_{n}(u) d u=b_{n, 1} C_{k}^{\alpha}(1)+\ldots+b_{n, n} C_{k}^{\alpha}(n) ;
$$

then in $L^{2}[0, \infty[$,

$$
B_{k}(u)=\sum_{n \geq 1} \gamma_{k}^{\alpha}(n) q_{n}(u) \quad(k \in \mathbb{Z})
$$

Using (2.4), we have in $L^{2}(\mathbb{R})$,

$$
g_{k}(\omega)=\sum_{n \geq 1} \gamma_{k}^{\alpha}(n)\left\{\psi_{n}(\omega)+\psi_{n}\left(\frac{2 \pi}{T} k-\omega\right)\right\} \quad(k \in \mathbb{Z})
$$


where

$$
\psi_{n}(\omega)=\frac{1}{2 \pi} \int_{0}^{\infty} \exp (-i \omega u) q_{n}(u) d u .
$$

Further, we will use (5.4) and (5.5) to find estimators of $g_{k}(\omega)$ and $B_{k}(u)$. First, we study kernel estimators of $g_{k}(\omega)$.

Let $H(\omega)$ be an even, absolutely integrable function, with $\int_{-\infty}^{\infty} H(\omega) d \omega$ $=1$. Let $M_{N}$ be a sequence of nonnegative numbers so that $\lim _{N \rightarrow \infty} M_{N}$ $=\infty$. Consider $W_{N}(\omega)=M_{N} H\left(M_{N} \omega\right), h(t)=\int_{-\infty}^{\infty} H(\omega) \exp (i \omega t) d t$ and $w_{N}(t)=h\left(t / M_{N}\right)$. Assuming that we get a sample $X\left(t_{1}\right), \ldots, X\left(t_{N}\right)$, we estimate $g_{k}(\omega)$ by the following estimators:

$$
\begin{aligned}
g_{k}^{(1)}(N, \omega)= & \frac{1}{2 \pi \beta N} \sum_{n=1}^{N-1} \sum_{l=1}^{N-n} w_{N}\left(t_{l+n}-t_{l}\right) X\left(t_{l+n}\right) X\left(t_{l}\right) \exp \left(-i \frac{2 \pi}{T} k t_{l}\right) \\
& \times\left\{\exp \left(i\left(\omega-\frac{2 \pi}{T} k\right)\left(t_{l+n}-t_{l}\right)\right)+\exp \left(-i \omega\left(t_{l+n}-t_{l}\right)\right)\right\}
\end{aligned}
$$

and

$$
\begin{aligned}
g_{k}^{(2)}(N, \omega)= & \frac{1}{2 \pi \beta N} \sum_{n=1}^{M_{N}} \sum_{l=1}^{N-n} X\left(t_{l+n}\right) X\left(t_{l}\right) \exp \left(-i \frac{2 \pi}{T} k t_{l}\right) \\
& \times\left\{\exp \left(i\left(\omega-\frac{2 \pi}{T} k\right)\left(t_{l+n}-t_{l}\right)\right)+\exp \left(-i \omega\left(t_{l+n}-t_{l}\right)\right)\right\} .
\end{aligned}
$$

Asymptotic properties of $g_{k}^{(1)}(N, \omega)$. We have

$$
\begin{aligned}
g_{k}^{(1)}(N, \omega)= & \frac{1}{4 \pi \beta N} \int_{-\infty}^{\infty} W_{N}(u) \sum_{n=1}^{N-1} \sum_{l=1}^{N-n} X\left(t_{l+n}\right) X\left(t_{l}\right) \exp \left(-i \frac{2 \pi}{T} k t_{l}\right) \\
& \times\left\{\exp \left(i\left(\omega+u-\frac{2 \pi}{T} k\right)\left(t_{l+n}-t_{l}\right)\right)\right. \\
& \left.+\exp \left(-i(\omega+u)\left(t_{l+n}-t_{l}\right)\right)\right\} d u \\
& +\frac{1}{4 \pi \beta N} \int_{-\infty}^{\infty} W_{N}(u) \sum_{n=1}^{N-1} \sum_{l=1}^{N-n} X\left(t_{l+n}\right) X\left(t_{l}\right) \exp \left(-i \frac{2 \pi}{T} k t_{l}\right) \\
& \times\left\{\exp \left(i\left(\omega-u-\frac{2 \pi}{T} k\right)\left(t_{l+n}-t_{l}\right)\right)\right. \\
= & I_{1}+I_{2} .
\end{aligned}
$$


Setting $\lambda=\omega+u$ (resp. $\lambda=\omega-u)$ in $I_{1}$ (resp. $I_{2}$ ) and using the evenness of $W_{N}$, we notice that $I_{1}=I_{2}$, therefore

$$
\begin{aligned}
g_{k}^{(1)}(N, \omega)= & \frac{1}{2 \pi \beta N} \int_{-\infty}^{\infty} W_{N}(\omega-\lambda) \sum_{n=1}^{N-1} \sum_{l=1}^{N-n} X\left(t_{l+n}\right) X\left(t_{l}\right) \exp \left(-i \frac{2 \pi}{T} k t_{l}\right) \\
& \times\left\{\exp \left(i\left(\lambda-\frac{2 \pi}{T} k\right)\left(t_{l+n}-t_{l}\right)\right)+\exp \left(-i \lambda\left(t_{l+n}-t_{l}\right)\right)\right\} d \lambda .
\end{aligned}
$$

We notice that if $\omega \neq 0$ then

$$
\left|\sum_{n=1}^{N} \phi(\omega)^{n}\right|=\left|\phi(\omega) \frac{\phi(\omega)^{N}-1}{\phi(\omega)-1}\right| \leq 2\left|\frac{\phi(\omega)}{\phi(\omega)-1}\right| \leq \frac{2 \beta}{|\omega|} .
$$

Theorem 5.1. If $X(t)$ is a $P C$ process with

$$
\int_{-\infty}^{\infty}\left(\sum_{j \neq k} \frac{\left|B_{j}(u)\right|}{|j-k|}+\left|B_{k}(u)\right|\right) d u<\infty
$$

then

$$
E\left\{g_{k}^{(1)}(N, \omega)\right\}=\int_{-\infty}^{\infty} W_{N}(\omega-\lambda) g_{k}(\lambda) d \lambda+o(1) .
$$

Proof. We have $E\left\{g_{k}^{(1)}(N, \omega)\right\}=\int_{-\infty}^{\infty} W_{N}(\omega-\lambda) I(\lambda) d \lambda$ where

$$
\begin{aligned}
I(\lambda)= & \frac{1}{2 \pi \beta N} E\left(\sum _ { n = 1 } ^ { N - 1 } \sum _ { l = 1 } ^ { N - n } \left\{\exp \left(i\left(\lambda-\frac{2 \pi}{T} k\right)\left(t_{l+n}-t_{l}\right)\right)\right.\right. \\
& \left.\left.+\exp \left(-i \lambda\left(t_{l+n}-t_{l}\right)\right)\right\} X\left(t_{l+n}\right) X\left(t_{l}\right) \exp \left(-i \frac{2 \pi}{T} k t_{l}\right)\right) \\
= & \frac{1}{2 \pi \beta N} \sum_{n=1}^{N-1} \sum_{l=1}^{N-n} \int_{0}^{\infty} \int_{0}^{\infty} \sum_{j \in \mathbb{Z}} B_{j}(u) \exp \left(i \frac{2 \pi}{T} j t\right) \exp \left(-i \frac{2 \pi}{T} k t\right) \\
& \times\left\{\exp \left(i\left(\lambda-\frac{2 \pi}{T} k\right) u\right)+\exp (-i \lambda u)\right\} f_{n}(u) f_{l}(t) d u d t .
\end{aligned}
$$

Since

$$
\int_{-\infty}^{\infty}\left(\sum_{j \neq k} \frac{\left|B_{j}(u)\right|}{|j-k|}+\left|B_{k}(u)\right|\right) d u<\infty
$$

(using Fubini's theorem), we have

$$
I(\lambda)=\frac{1}{2 \pi \beta N} \sum_{n=1}^{N-1} \int_{0}^{\infty} \sum_{j \in \mathbb{Z}} B_{j}(u)\left\{\exp \left(i\left(\lambda-\frac{2 \pi}{T} k\right) u\right)\right.
$$




$$
\begin{aligned}
& +\exp (-i \lambda u)\} f_{n}(u)\left(\sum_{l=1}^{N-n} \int_{0}^{\infty} \exp \left(i \frac{2 \pi}{T}(j-k) t\right) f_{l}(t) d t\right) d u \\
= & I_{1}(\lambda)+I_{2}(\lambda)
\end{aligned}
$$

where

$$
\begin{aligned}
I_{1}(\lambda)=\frac{1}{2 \pi \beta N} \sum_{n=1}^{N-1}(N-n) \int_{0}^{\infty} B_{k}(u)\left\{\exp \left(i\left(\lambda-\frac{2 \pi}{T} k\right) u\right)\right. \\
+\exp (-i \lambda u)\} f_{n}(u) d u
\end{aligned}
$$

and

$$
\begin{aligned}
I_{2}(\lambda)=\frac{1}{2 \pi \beta N} \sum_{n=1}^{N-1} \int_{0}^{\infty} & \left(\sum _ { j \neq k } B _ { j } ( u ) \left\{\exp \left(i\left(\lambda-\frac{2 \pi}{T} k\right) u\right)\right.\right. \\
& \left.+\exp (-i \lambda u)\} \sum_{l=1}^{N-n} \phi\left(\frac{2 \pi}{T}(j-k)\right)^{l}\right) f_{n}(u) d u
\end{aligned}
$$

Using (5.6) and Lemma 1 of [6], we have

$$
\left|I_{2}(\lambda)\right| \leq \frac{4}{2 \pi \beta N} \sum_{n=1}^{N-1} \int_{0}^{\infty}\left(\sum_{j \neq k}\left|B_{j}(u)\right| \frac{\beta}{\frac{2 \pi}{T}|j-k|}\right) f_{n}(u) d u=O\left(\frac{1}{N}\right)
$$

where the $O(1 / N)$ term is uniform in $\lambda$. Put

$$
e_{n}^{k}(\lambda)=\int_{0}^{\infty} B_{k}(u)\left\{\exp \left(i\left(\lambda-\frac{2 \pi}{T} k\right) u\right)+\exp (-i \lambda u)\right\} f_{n}(u) d u .
$$

Then

$$
\begin{aligned}
\frac{1}{2 \pi \beta} \sum_{n=1}^{\infty} e_{n}^{k}(\lambda)=\frac{1}{2 \pi} \int_{0}^{\infty} B_{k}(u)\left\{\exp \left(i\left(\lambda-\frac{2 \pi}{T}\right) u\right)\right. \\
+\exp (-i \lambda u)\} d u=g_{k}(\lambda)
\end{aligned}
$$

and therefore

$$
\begin{aligned}
I_{1}(\lambda) & =\frac{1}{2 \pi \beta} \sum_{n=1}^{N-1}\left(1-\frac{n}{N}\right) e_{n}^{k}(\lambda) \\
& =g_{k}(\lambda)-\frac{1}{2 \pi \beta} \sum_{n=N}^{\infty} e_{n}^{k}(\lambda)-\frac{1}{2 \pi \beta N} \sum_{n=1}^{N-1} n e_{n}^{k}(\lambda) .
\end{aligned}
$$

Since $\left|e_{n}^{k}(\lambda)\right| \leq 2 \int_{0}^{\infty}\left|B_{k}(u)\right| f_{n}(u) d u$, the series $\sum_{n \geq 1} e_{n}^{k}(\lambda)$ converges uniformly (Lemma 1 of [6]); so $\sum_{n \geq N} e_{n}^{k}(\lambda)$ and $\frac{1}{N} \sum_{n=1}^{N-1} n e_{n}^{k}(\lambda)$ (Kronecker's 
lemma) converge uniformly to 0 . Finally,

$$
\begin{aligned}
E\left\{g_{k}^{(1)}(N, \omega)\right\} & =\int_{-\infty}^{\infty} W_{N}(\omega-\lambda)\left[g_{k}(\lambda)+o(1)+O(1 / N)\right] d \lambda \\
& =\int_{-\infty}^{\infty} W_{N}(\omega-\lambda) g_{k}(\lambda) d \lambda+o(1) .
\end{aligned}
$$

Corollary 5.1. If $X(t)$ is a $P C$ process with

$$
\int_{-\infty}^{\infty}\left(\sum_{j \neq k} \frac{\left|B_{j}(u)\right|}{|j-k|}+\left|B_{k}(u)\right|\right) d u<\infty
$$

and $\int_{0}^{\infty}\left|u B_{k}(u)\right| d u \leq \infty$, then

$$
E\left\{g_{k}^{(1)}(N, \omega)\right\}=\int_{-\infty}^{\infty} W_{N}(\omega-\lambda) g_{k}(\lambda) d \lambda+O\left(\frac{1}{N}\right) .
$$

Proof. We have

$$
I_{1}=g_{k}(\lambda)-\frac{1}{2 \pi \beta} \sum_{n \geq N} e_{n}^{k}(\lambda)-\frac{1}{2 \pi \beta N} \sum_{n=1}^{N-1} n e_{n}^{k}(\lambda)
$$

and using Lemma 1(ii) of [6], we have

$$
\left|\sum_{n \geq N} e_{n}^{k}(\lambda)\right| \leq \frac{2}{N} \sum_{n=N}^{\infty} n \int_{0}^{\infty}\left|B_{k}(u)\right| f_{n}(u) d u=O\left(\frac{1}{N}\right)
$$

and

$$
\left|\frac{1}{2 \pi \beta N} \sum_{n=1}^{N-1} n e_{n}^{k}(\lambda)\right| \leq \frac{1}{N} \sum_{n=1}^{N-1} n \int_{0}^{\infty}\left|B_{k}(u)\right| f_{n}(u) d u=O\left(\frac{1}{N}\right) .
$$

We notice that the $O$ term is independent of $\lambda$, which gives the result.

Corollary 5.2. If $X(t)$ is a $P C$ process with

$$
\int_{-\infty}^{\infty}\left(\sum_{j \neq k} \frac{\left|B_{j}(u)\right|}{|j-k|}+\left|B_{k}(u)\right|\right) d u<\infty
$$

then $g_{k}^{(1)}$ is an asymptotically unbiased estimator.

Pr o of. By $(2.1)$ we see that $g_{k}(\omega)$ is a continuous and bounded function. On the other hand,

$$
E\left\{g_{k}^{(1)}(N, \omega)\right\}=\int_{-\infty}^{\infty} W_{N}(\omega-\lambda) g_{k}(\lambda) d \lambda+o(1)
$$




$$
\begin{aligned}
& =\int_{-\infty}^{\infty} M_{N} H\left(M_{N}(\omega-\lambda)\right) g_{k}(\lambda) d \lambda+o(1) \\
& =\int_{-\infty}^{\infty} H(u) g_{k}\left(\omega-\frac{u}{M_{N}}\right) d u+o(1)
\end{aligned}
$$

and we use Lebesgue's theorem to conclude.

COROLlary 5.3. In addition to the hypothesis of Theorem 5.1, let $q$ be a positive integer such that $u^{q} B_{k}(u) \in L^{1}[0, \infty[$ and assume $h(t)$ is $q$ times differentiable with bounded derivatives. Then

$$
E\left\{g_{k}^{(1)}(N, \omega)\right\}=g_{k}(\omega)+\sum_{l=1}^{q-1} \frac{i^{l} h^{(l)}(0)}{M_{N}^{l}} g_{k}^{(l)}(\omega)+O\left(\frac{1}{M_{N}^{q}}\right)+O\left(\frac{1}{N}\right)
$$

where $f^{(l)}$ is the derivative of $f$ of order $l$.

The proof is identical to the proof of Corollary 1.2 of [5], p. 175 .

To prove the consistency of the estimator $g_{k}^{(1)}(N, \lambda)$, we make the following assumptions:

$\left(\mathcal{H}_{1}\right) \quad$ i) $\int_{-\infty}^{\infty}\left(\sum_{j \neq k} \frac{\left|B_{j}(u)\right|}{|j-k|}+\left|B_{k}(u)\right|\right) d u<\infty, \quad \int_{0}^{\infty}\left|u B_{k}(u)\right| d u<\infty$,

(ii) $\sum_{j \in \mathbb{Z}}\left|B_{j}(0)\right| \int_{0}^{\infty}\left|B_{j}(u)\right| d u<\infty, \quad \sum_{j \in \mathbb{Z}}\left|B_{j}(0)\right|^{2}<\infty$.

$\left(\mathcal{H}_{2}\right)$ For $j \geq 0$, there exists $h_{j}(u)$ such that

(i) $h_{j}(u)$ is a continuous, even, nonnegative, integrable and nondecreasing function on $[0, \infty[$,

(ii) $\left|B_{j}(u)\right| \leq h_{j}(u)$,

(iii) $\int_{0}^{\infty} \int_{0}^{\infty}\left(\sum_{j_{1}+j_{2} \neq 0} \frac{1}{\left|j_{1}+j_{2}\right|} h_{j_{1}}\left(u_{1}\right) h_{j_{2}}\left(u_{2}\right)\right.$

$$
\left.+\sum_{j_{1}+j_{2}=0} h_{j_{1}}\left(u_{1}\right) h_{j_{2}}\left(u_{2}\right)\right) d u_{1} d u_{2}<\infty
$$

(iv) $\int_{0}^{\infty} \int_{0}^{\infty}\left(\sum_{j_{1}+j_{2} \neq 0} \frac{1}{\left|j_{1}+j_{2}\right|} h_{j_{1}}\left(u_{1}\right) h_{j_{2}}\left(u_{2}\right)\right.$

$$
\left.+\sum_{j_{1}+j_{2}=0} h_{j_{1}}\left(u_{1}\right) h_{j_{2}}\left(u_{2}\right)\right)\left(u_{1}+u_{2}\right) d u_{1} d u_{2}<\infty .
$$

$\left(\mathcal{H}_{3}\right) \quad X(t)$ is a PC process up to order 4 and for $j \in \mathbb{Z}$, there exist nonnegative functions $q_{j}^{1}, q_{j}^{2}, q_{j}^{3}$ so that 


$$
\left|K_{j}\left(u_{1}, u_{2}, u_{3}\right)\right| \leq \prod_{i=1}^{3} q_{j}^{i}\left(u_{i}\right),
$$

$\int_{0}^{\infty} \int_{0}^{\infty} \int_{0}^{\infty}\left(\sum_{j \neq k} \frac{q_{j}^{1}\left(u_{1}\right) q_{j}^{2}\left(u_{2}\right) q_{j}^{3}\left(u_{3}\right)}{|j-k|}+q_{k}^{1}\left(u_{1}\right) q_{k}^{2}\left(u_{2}\right) q_{k}^{3}\left(u_{3}\right)\right) d u_{1} d u_{2} d u_{3}<\infty$.

We also assume that the sequence $M_{N}$ satisfies $\lim _{N \rightarrow \infty} M_{N} / N=0$.

Let

$$
\begin{aligned}
p(l, n, k, \lambda)=\left(\exp \left(i\left(\lambda-\frac{2 \pi}{T} k\right)\left(t_{l+n}-t_{l}\right)\right)\right. & \\
& \left.+\exp \left(-i \lambda\left(t_{l+n}-t_{l}\right)\right)\right) \exp \left(-i \frac{2 \pi}{T} k t_{l}\right) .
\end{aligned}
$$

Then

$$
\begin{aligned}
\operatorname{var}\{ & \left.g_{k}^{(1)}(N, \omega)\right\} \\
& =\int_{-\infty}^{\infty} \int_{-\infty}^{\infty} W_{N}(\omega-\lambda) W_{N}(\omega-\mu) \operatorname{cov}\left\{J_{k}(N, \lambda) ; J_{k}(N, \mu)\right\} d \lambda d \mu
\end{aligned}
$$

where

We have

$$
J_{k}(N, \lambda)=\frac{1}{2 \pi \beta N} \sum_{n=1}^{N-1} \sum_{l=1}^{N-n} X\left(t_{l+n}\right) X\left(t_{l}\right) p(l, n, k, \lambda) .
$$

$$
\begin{aligned}
E\left\{J_{k}(N, \lambda) \overline{J_{k}(N, \mu)}\right\} & \\
=\frac{1}{(2 \pi \beta N)^{2}} \sum_{n_{1}, n_{2}=1}^{N-1} & \sum_{l_{1}=1}^{N-n_{1}} \sum_{l_{2}=1}^{N-n_{2}} E\left(p\left(l_{1}, n_{1}, k, \lambda\right) \overline{p\left(l_{2}, n_{2}, k, \mu\right)}\right. \\
& \left.\times E\left\{X\left(t_{l_{1}+n_{1}}\right) X\left(t_{l_{1}}\right) X\left(t_{l_{2}+n_{2}}\right) X\left(t_{l_{2}}\right) \mid\left(t_{n}\right)\right\}\right),
\end{aligned}
$$

so $\operatorname{cov}\left\{J_{k}(N, \lambda) ; J_{k}(N, \mu)\right\}=\sum_{i=1}^{4} U_{N, i}(k, \lambda, \mu)-E\left\{J_{k}(N, \lambda)\right\} E\left\{\overline{J_{k}(N, \mu)}\right\}$ where

(5.8a) $\quad U_{N, 1}(k, \lambda, \mu)$

$$
\begin{aligned}
=\frac{1}{(2 \pi \beta N)^{2}} \sum_{n_{1}, n_{2}=1}^{N-1} \sum_{\substack{\left.l_{1}, l_{2} \in R \\
\\
\times B\left(t_{l_{1}}, t_{l_{1}+n_{1}}-t_{l_{1}}\right) B\left(t_{l_{2}}, t_{l_{2}+n_{2}}-t_{l_{2}}\right)\right),}} E\left(p\left(l_{1}, n_{1}, k, \lambda\right) \overline{p\left(l_{2}, n_{2}, k, \mu\right)}\right.
\end{aligned}
$$

(5.8b) $\quad U_{N, 2}(k, \lambda, \mu)$

$$
\begin{aligned}
& =\frac{1}{(2 \pi \beta N)^{2}} \sum_{n_{1}, n_{2}=1}^{N-1} \sum_{l_{1}, l_{2} \in R} E\left(p\left(l_{1}, n_{1}, k, \lambda\right) \overline{p\left(l_{2}, n_{2}, k, \mu\right)}\right. \\
& \left.\times B\left(t_{l_{1}}, t_{l_{2}}-t_{l_{1}}\right) B\left(t_{l_{1}+n_{1}}, t_{l_{2}+n_{2}}-t_{l_{1}+n_{1}}\right)\right),
\end{aligned}
$$


(5.8c) $\quad U_{N, 3}(k, \lambda, \mu)$

$$
\begin{aligned}
=\frac{1}{(2 \pi \beta N)^{2}} \sum_{n_{1}, n_{2}=1}^{N-1} \sum_{l_{1}, l_{2} \in R} E\left(p\left(l_{1}, n_{1}, k, \lambda\right) \overline{p\left(l_{2}, n_{2}, k, \mu\right)}\right. \\
\left.\times B\left(t_{l_{1}}, t_{l_{2}+n_{2}}-t_{l_{1}}\right) B\left(t_{l_{2}}, t_{l_{1}+n_{1}}-t_{l_{2}}\right)\right),
\end{aligned}
$$

(5.8d) $\quad U_{N, 4}(k, \lambda, \mu)$

$$
\begin{aligned}
& =\frac{1}{(2 \pi \beta N)^{2}} \sum_{n_{1}, n_{2}=1}^{N-1} \sum_{l_{1}, l_{2} \in R} E\left(p\left(l_{1}, n_{1}, k, \lambda\right) \overline{p\left(l_{2}, n_{2}, k, \mu\right)}\right. \\
& \left.\times K\left(t_{l_{1}}, t_{l_{1}+n_{1}}-t_{l_{1}}, t_{l_{2}+n_{2}}-t_{l_{1}}, t_{l_{2}}-t_{l_{1}}\right)\right),
\end{aligned}
$$

with $R=\left\{\left(l_{1}, l_{2}\right) \in \mathbb{N}^{2}: 1 \leq l_{1} \leq N-n_{1}, 1 \leq l_{2} \leq N-n_{2}\right\}$.

We give here the main results, the proofs are in the appendix.

Proposition 5.3. (a) Under assumptions $\left(\mathcal{H}_{1}\right)$ and $\left(\mathcal{H}_{2}\right)$,

$$
U_{N, 1}(k, \lambda, \mu)-E\left\{J_{k}(N, \lambda)\right\} E\left\{\overline{J_{k}(N, \mu)}\right\}=O(1 / N)
$$

and

$$
U_{N, 3}(k, \lambda, \mu)=O(1 / N)
$$

(b) Under assumption $\left(\mathcal{H}_{3}\right)$,

$$
U_{N, 4}(k, \lambda, \mu)=O(1 / N) .
$$

The $O$ terms are uniform in $\lambda$ and $\mu$.

Proposition 5.4. Under assumptions $\left(\mathcal{H}_{1}\right)$ and $\left(\mathcal{H}_{2}\right)$, if $H$ is a bounded function with a bounded derivative, then

$$
U_{N, 2}(k, \lambda, \mu)=O\left(M_{N} / N\right)
$$

uniformly in $\lambda$ and $\mu$.

THEOREM 5.2. If $H$ is a bounded function with a bounded derivative, then under assumptions $\left(\mathcal{H}_{1}\right),\left(\mathcal{H}_{2}\right)$ and $\left(\mathcal{H}_{3}\right)$, we have for $\lambda, \mu \in \mathbb{R}$ and $j, k \in \mathbb{Z}$,

$$
\operatorname{var}\left\{g_{k}^{(1)}(N, \mu)\right\}=O\left(M_{N} / N\right) .
$$

Proof. We just have to apply Propositions 5.3 and 5.4 and use (5.7) and $(5.8 \mathrm{a})-(5.8 \mathrm{~d})$.

Asymptotic properties of $g_{k}^{(2)}(N, \omega)$

Theorem 5.3. If $X(t)$ is a $P C$ process with

$$
\int_{-\infty}^{\infty}\left(\sum_{j \neq k} \frac{\left|B_{j}(u)\right|}{|j-k|}+\left|B_{k}(u)\right|\right) d u<\infty
$$


then

$$
E\left\{g_{k}^{(2)}(N, \omega)\right\}=g_{k}(\omega)+o(1)
$$

where the o(1) term is uniform in $\omega$.

Proof. Using previous expressions, we get

$$
E\left\{g_{k}^{(2)}(N, \omega)\right\}=\frac{N-n}{2 \pi \beta N} \sum_{n=1}^{M_{N}} e_{n}^{k}(\omega)+O\left(\frac{1}{N}\right) .
$$

On the other hand, $\sum_{n=1}^{\infty} e_{n}^{k}(\omega)=2 \pi \beta g_{k}(\omega)$, therefore

$E\left\{g_{k}^{(2)}(N, \omega)\right\}=g_{k}(\omega)-\frac{1}{2 \pi \beta} \sum_{n=M_{N}+1}^{\infty} e_{n}^{k}(\omega)-\frac{1}{2 \pi \beta N} \sum_{n=1}^{M_{N}} n e_{n}^{k}(\omega)+O\left(\frac{1}{N}\right)$.

Since $\left|\sum_{n=M_{N}+1}^{\infty} e_{n}^{k}(\omega)\right|=o(1)$ uniformly in $\omega$ as $N \rightarrow \infty$, therefore

$$
\left|\frac{1}{N} \sum_{n=1}^{M_{N}} n e_{n}^{k}(\omega)\right| \leq \frac{M_{N}}{N} \frac{1}{M_{N}}\left|\sum_{n=1}^{M_{N}} n e_{n}^{k}(\omega)\right|=\frac{M_{N}}{N} o(1),
$$

which gives the result.

Corollary 5.4. If $X(t)$ is a $P C$ process with

$$
\int_{-\infty}^{\infty}\left(\sum_{j \neq k} \frac{\left|B_{j}(u)\right|}{|j-k|}+\left|B_{k}(u)\right|\right) d u<\infty
$$

and $\int_{0}^{\infty} u\left|B_{k}(u)\right| d u<\infty$, then

$$
E\left\{g_{k}^{(2)}(N, \omega)\right\}=g_{k}(\omega)+O\left(1 / M_{N}\right) .
$$

Pr o of. In this case the series $n e_{n}^{k}(\omega)$ converges uniformly, therefore

$$
\left|\sum_{n=M_{N}+1}^{\infty} e_{n}^{k}(\omega)\right| \leq \frac{1}{M_{N}} \sum_{n=M_{N}+1}^{\infty} n\left|e_{n}^{k}(\omega)\right|=\frac{1}{M_{N}} o(1)
$$

and $\left|\frac{1}{N} \sum_{n=1}^{M_{N}} n e_{n}^{k}(\omega)\right|=O(1 / N)$, which gives the result.

To prove the consistency of the estimator $g_{k}^{(2)}(N, \omega)$, we make the following assumptions:

$$
\begin{aligned}
\left(\mathcal{H}_{1}^{\prime}\right) \text { (i) } \int_{0}^{\infty}\left(\sum_{j_{1} \neq-j_{2}} \frac{\left|B_{j_{1}}\left(u_{1}\right) B_{j_{2}}\left(u_{2}\right)\right|}{\left|j_{1}+j_{2}\right|}\right) d u_{1} d u_{2}+\int_{0}^{\infty}\left(\sum_{j \in \mathbb{Z}}\left|B_{j}(u)\right|^{2}\right) d u<\infty \\
\text { (ii) } \int_{0}^{\infty}\left(\sum_{j_{1} \neq-j_{2}} \frac{\left|B_{j_{1}}\left(u_{1}\right) B_{j_{2}}\left(u_{2}\right)\right|}{\left|j_{1}+j_{2}\right|}\right)\left(u_{1}+u_{2}\right) d u_{1} d u_{2} \\
+\int_{0}^{\infty}\left(\sum_{j \in \mathbb{Z}}\left|B_{j}(u)\right|^{2}\right) u d u<\infty
\end{aligned}
$$


$\left(\mathcal{H}_{2}^{\prime}\right) \quad X(t)$ is a PC process up to order 4 and for all $j \in \mathbb{Z}$, we have

$$
\left|K_{j}\left(u_{1}, u_{2}, u_{3}\right)\right| \leq h_{j}\left(u_{1}, u_{2}, u_{3}\right)
$$

where $h_{j}$ is a nondecreasing function on $[0, \infty[$, even in each variable and such that

$$
\sum_{j \in \mathbb{Z}} \int_{0}^{\infty} \frac{h_{j}(0, u, 0)}{|j-k|} d u<\infty, \quad \sum_{j \in \mathbb{Z}} h_{j}(0,0,0)<\infty .
$$

Let

$$
\begin{array}{r}
\Gamma_{k}(n, N, \omega)=\frac{1}{N} \sum_{l=1}^{N-n}\left\{\operatorname { e x p } \left(i\left(\omega-\frac{2 \pi}{T} k\right)\right.\right. \\
\left.\left.\left(t_{l+n}-t_{l}\right)\right)+\exp \left(-i \omega\left(t_{l+n}-t_{l}\right)\right)\right\} \\
\times X\left(t_{l+n}\right) X\left(t_{l}\right) \exp \left(-i \frac{2 \pi}{T} k t_{l}\right) .
\end{array}
$$

Then

therefore

$$
E\left\{\Gamma_{k}(n, N, \omega)\right\}=\frac{N-n}{N} e_{n}^{k}(\omega)+\frac{1}{N} o(1)
$$

$$
\left|E\left\{\Gamma_{k}(n, N, \omega)\right\}\right|^{2}=\left(\frac{N-n}{N}\right)^{2}\left|e_{n}^{k}(\omega)\right|^{2}+\frac{1}{N} o(1) .
$$

Proposition 5.5. Under assumptions $\left(\mathcal{H}_{1}^{\prime}\right)$ and $\left(\mathcal{H}_{2}^{\prime}\right)$, if $M_{N}^{2} / N \rightarrow 0$, then

$$
\operatorname{var}\left\{\Gamma_{k}(n, N, \omega)\right\}=O(1 / N) .
$$

THEOREM 5.4. Under assumptions $\left(\mathcal{H}_{1}^{\prime}\right)$ and $\left(\mathcal{H}_{2}^{\prime}\right)$, if $M_{N}^{2} / N \rightarrow 0$, then

$$
\operatorname{var}\left\{g_{k}^{(2)}(N, \omega)\right\}=O\left(M_{N}^{2} / N\right) .
$$

Pr o of. Using the above proposition, we have

$$
\begin{aligned}
{\left[\operatorname{var}\left\{g_{k}^{(2)}(N, \omega)\right\}\right]^{1 / 2} } & \leq \frac{1}{2 \pi \beta} \sum_{n=1}^{M_{N}}\left[\operatorname{var}\left\{\Gamma_{k}(n, N, \omega)\right\}\right]^{1 / 2} \\
& \leq \frac{M_{N}}{2 \pi \beta} O\left(\frac{1}{N^{1 / 2}}\right),
\end{aligned}
$$

which gives the result.

Estimation by means of an orthogonal series with truncation. Earlier, we showed that there exists a complete orthonormal system $\left\{q_{n}(u): n \geq 1\right\}$ on $L^{2}\left[0, \infty\left[\right.\right.$ such that $q_{n}(u)=\sum_{l=1}^{n} b_{n, l} f_{l}(u)$ where $b_{n, l}=(2 / \beta)^{1 / 2}(-2)^{l-1} \mathcal{C}_{n-1}^{l-1}$. We have $g_{k}(\omega)=\sum_{n \geq 1} \gamma_{k}(n) Q_{n}^{k}(\omega)$ where

$$
Q_{n}^{k}(\omega)=\frac{1}{2 \pi} \int_{0}^{\infty}\left\{\exp \left(i\left(\omega-\frac{2 \pi}{T} k\right) u\right)+\exp (-i \omega u)\right\} q_{n}(u) d u
$$


and

$$
\gamma_{k}(n)=\int_{0}^{\infty} B_{k}(u) q_{n}(u) d u=\sum_{l=1}^{n} b_{n, l} C_{k}^{\alpha}(l) .
$$

As in F. Messaci's thesis [7], for all nonnegative integers $n$ and for all integers $k$,

$$
\left|Q_{n}^{k}(\omega)\right| \leq \frac{1}{\pi}\left(\frac{2}{\beta}\right)^{1 / 2} .
$$

Assuming that we get a sample $\left\{X\left(t_{k}\right): k=1, \ldots, N\right\}$, using (5.1), we estimate $C_{k}^{\alpha}(n)$ by

$$
\widehat{C}_{k}^{\alpha}(n, N)=\frac{1}{N} \sum_{l=1}^{N-n} X\left(t_{l+n}\right) X\left(t_{l}\right) \exp \left(-i \frac{2 \pi}{T} k t_{l}\right) .
$$

It is natural to estimate $\gamma_{k}(n)$ using (5.3), by

$$
\widehat{\gamma}_{k}(n, N)=\sum_{l=1}^{n} b_{n, l} \widehat{C}_{k}^{\alpha}(l, N) .
$$

Then we estimate $g_{k}(\omega)$ by

$$
g_{k}^{(3)}(N, \omega)=\sum_{n=1}^{M_{N}} Y_{n}(N) \widehat{\gamma}_{k}(n, N) Q_{n}^{k}(\omega)
$$

where $M_{N}$ is the integer part of $\frac{b}{2 \alpha} \ln (N)+1$ and

$$
Y_{n}(N)=h\left(\frac{\exp (n \alpha)}{N^{b}}\right), \quad \alpha>0,0<b<\frac{\alpha}{\ln 3},
$$

where $h$ is a function satisfying

(i) $|h(u)| \leq 1 \forall u \in \mathbb{R}$,

(ii) $|1-h(u)| \leq|u| \forall u \in \mathbb{R}$.

Let $A C^{r}[0, \infty$ [ be the class of functions which are $r$ times differentiable with absolutely continuous derivatives. To study the asymptotic behavior of $g_{k}^{(3)}(N, \omega)$, we make the following assumption:

$\left(\mathcal{H}_{1}^{\prime \prime}\right) \quad$ There exists $r>2$ so that for $u \geq 0$ and an integer $k$, we have

(i) $B_{k}(u) \in A C^{r-1}[0, \infty[$,

(ii) $u^{r / 2} B_{k}^{(l)}(u) \in L^{2}[0, \infty[, \quad l=0,1, \ldots, r$.

We follow F. Messaci's method [7].

LEMma 5.3. Under assumption $\left(\mathcal{H}_{1}^{\prime \prime}\right)$, for all integers $n \geq 0$ and $k$,

(i) $\left|\gamma_{k}(n)\right| \leq A_{n}^{k}(r) n^{-r / 2}$,

(ii) $g_{k}(\omega)=\sum_{n=1}^{\infty} \gamma_{k}(n) Q_{n}^{k}(\omega)$ uniformly on $\mathbb{R}$. 
Proposition 5.6. Under assumptions $\left(\mathcal{H}_{1}^{\prime}\right)$ and $\left(\mathcal{H}_{2}^{\prime}\right)$,

$$
\begin{gathered}
E\left\{\widehat{C}_{k}^{\alpha}(n, N)\right\}=\frac{N-n}{N} C_{k}^{\alpha}(n)+O\left(\frac{1}{N}\right), \\
\operatorname{var}\left\{\widehat{C}_{k}^{\alpha}(n, N)\right\} \leq \frac{A_{2}}{N} .
\end{gathered}
$$

The proof of this proposition is analogous to the proofs of Proposition 5.5 and Theorem 5.4.

Proposition 5.7. Under assumptions $\left(\mathcal{H}_{1}^{\prime}\right)$ and $\left(\mathcal{H}_{2}^{\prime}\right)$,

$$
\begin{aligned}
E\left\{\widehat{\gamma}_{k}(n, N)\right\}= & \gamma_{k}(n)-\frac{1}{N}\left\{n \gamma_{k}(n)-(n-1) \gamma_{k}(n-1)\right\} \\
& +O\left(\frac{1}{N}\right) \sum_{l=1}^{n} b_{n, l}, \\
& \operatorname{var}\left\{\widehat{\gamma}_{k}(n, N)\right\} \leq A_{3} \frac{3^{n}}{N} .
\end{aligned}
$$

The proof of this proposition is analogous to the proof of Theorem 2 of [7], p. 25 .

THeOREM 5.5. If $X(t)$ satisfies assumptions $\left(\mathcal{H}_{1}^{\prime}\right),\left(\mathcal{H}_{2}^{\prime}\right)$ and $\left(\mathcal{H}_{1}^{\prime \prime}\right)$, then $g_{k}^{(3)}(N, \omega)$ is uniformly consistent, with

$$
E\left\{\left|g_{k}^{(3)}(N, \omega)-g_{k}(\omega)\right|^{2}\right\}=O\left(\frac{1}{\ln N}\right)^{r-2} .
$$

Proof. From the proof of Theorem 3 of [7], p. 27, we have

$$
E\left\{\left|g_{k}^{(3)}(N, \omega)-g_{k}(\omega)\right|^{2}\right\} \leq V_{k}(N, \omega)+\left|O\left(\frac{1}{N}\right)\right| \sum_{n=1}^{M_{N}}\left|\gamma_{k}(n) Q_{n}^{k}(\omega)\right| \sum_{l=1}^{n}\left|b_{n, l}\right|
$$

with $V_{k}(N, \omega)=O(1 / \ln N)^{r-2}$. On the other hand, we have

$$
\sum_{n=1}^{M_{N}}\left|\gamma_{k}(n) Q_{n}^{k}(\omega)\right| \sum_{l=1}^{n}\left|b_{n, l}\right| \leq \frac{2}{\pi \beta} A_{1}(r) \sum_{n=1}^{M_{N}} n^{-r / 2} \sum_{l=1}^{n} \mathcal{C}_{n-1}^{l-1} 2^{l-1}
$$

and

$$
\begin{aligned}
\frac{1}{N} \sum_{n=1}^{M_{N}}\left|\gamma_{k}(n) Q_{n}^{k}(\omega)\right| \sum_{l=1}^{n}\left|b_{n, l}\right| & \leq \frac{2}{\pi \beta} A_{1}(r) \sum_{n=1}^{M_{N}} 3^{n} \\
& \leq \frac{2}{\pi \beta} A_{1}(r) \frac{3}{2 N} 3^{M_{N}-1} \\
& \leq \frac{2}{\pi \beta} A_{1}(r) \frac{3}{2} N^{-\left(1-\frac{b}{2 \alpha} \ln 3\right)} .
\end{aligned}
$$


Therefore

$$
\frac{1}{N} \sum_{n=1}^{M_{N}}\left|\gamma_{k}(n) Q_{n}^{k}(\omega)\right| \sum_{l=1}^{n}\left|b_{n, l}\right| \leq O\left(N^{-q}\right)
$$

with $q=1-\frac{b}{2 \alpha} \ln 3>0$. Thus

$$
\frac{1}{N} \sum_{n=1}^{M_{N}}\left|\gamma_{k}(n) Q_{n}^{k}(\omega)\right| \sum_{l=1}^{n}\left|b_{n, l}\right|=O\left(\frac{1}{\ln N}\right)^{r-2} .
$$

Generalization to almost periodically correlated harmonizable and gaussian processes. If $X(t)$ is almost periodically correlated, then

$$
B(t, u)=E\{X(t+u) X(t)\}=\sum_{\alpha \in S(X)} B_{\alpha}(u) \exp (i \alpha t)
$$

where the set $S(X)$ is at most countable [1]. If $B_{\alpha} \in L^{1}(\mathbb{R})$ for some $\alpha$, we define the spectral density function $g_{\alpha}$ by

$$
g_{\alpha}(\omega)=\frac{1}{2 \pi} \int_{-\infty}^{\infty} B_{\alpha}(u) \exp (-i u \omega) d u .
$$

If we assume that $X(t)$ is harmonizable, gaussian and

$$
\int_{0}^{\infty}\left(\sum_{\lambda \in S(X)}\left|B_{\lambda}(u)\right|^{2}\right) d u<\infty
$$

then the above results are valid.

6. Examples of periodically correlated processes. We give here some examples of PC processes which will be used for simulations in a next paper.

Let $Y(t)$ be a stationary process with continuous autocovariance function $r_{Y}(u)$. Let $P(t)$ be a bounded periodic function with period $T$. Put

$$
X(t, \omega)=P(t) Y(t, \omega) .
$$

Then

We have

$$
B(t, u)=E\{X(t+u) X(t)\}=P(t+u) P(t) r_{Y}(u) .
$$

$$
\begin{aligned}
B_{k}(u) & =\frac{1}{T} r_{Y}(u) \int_{0}^{T} P(t+u) P(t) \exp \left(-i \frac{2 \pi}{T} k t\right) d t \\
& =r_{Y}(u) \sum_{n \in \mathbb{Z}} \exp \left(i \frac{2 \pi}{T} n u\right) p_{n} \bar{p}_{n-k}
\end{aligned}
$$

where

$$
p_{n}=\frac{1}{T} \int_{0}^{T} P(t) \exp \left(-\frac{2 \pi}{T} n t\right) d t
$$


1) If we choose $P(t)$ so that $p_{n}=0$ for $|n|>A$ and $Y(t)$ is a Markov gaussian process with $E Y(t)=0$ and autocovariance function $r_{Y}(u)=e^{-|u|}$, then $X(t)$ is a PC process which satisfies the assumptions of the above theorems.

2 ) If we choose $P(t)$ so that $\sum_{n \in \mathbb{Z}}\left|p_{n}\right|<\infty$ and $Y(t)$ as before, then we have the same conclusion as in 1).

Other examples can be found in [2].

7. Appendix. We follow and generalize Masry and Lui's method [6]. Let

$$
\begin{aligned}
R_{1} & :=\left\{\left(l_{1}, l_{2}\right) \in R: l_{1}<l_{1}+n_{1} \leq l_{2}<l_{2}+n_{2}\right\}, \\
R_{2} & :=\left\{\left(l_{1}, l_{2}\right) \in R: l_{1}<l_{2}<l_{1}+n_{1}<l_{2}+n_{2}\right\}, \\
R_{3} & :=\left\{\left(l_{1}, l_{2}\right) \in R: l_{1}=l_{2}<l_{1}+n_{1}<l_{2}+n_{2}\right\}, \\
R_{4} & :=\left\{\left(l_{1}, l_{2}\right) \in R: l_{1}<l_{2}<l_{1}+n_{1}=l_{2}+n_{2}\right\}, \\
R_{5} & :=\left\{\left(l_{1}, l_{2}\right) \in R: l_{1}<l_{2}<l_{2}+n_{2}<l_{1}+n_{1}\right\}, \\
R_{6} & :=\left\{\left(l_{1}, l_{2}\right) \in R: l_{1}=l_{2}<l_{1}+n_{1}=l_{2}+n_{2}\right\}, \\
R_{7} & :=\left\{\left(l_{1}, l_{2}\right) \in R: l_{2}<l_{2}+n_{2} \leq l_{1}<l_{1}+n_{1}\right\}, \\
R_{8} & :=\left\{\left(l_{1}, l_{2}\right) \in R: l_{2}<l_{1}<l_{2}+n_{2}<l_{1}+n_{1}\right\}, \\
R_{9} & :=\left\{\left(l_{1}, l_{2}\right) \in R: l_{2}=l_{1}<l_{2}+n_{2}<l_{1}+n_{1}\right\}, \\
R_{10} & :=\left\{\left(l_{1}, l_{2}\right) \in R: l_{2}<l_{1}<l_{2}+n_{2}<l_{1}+n_{1}\right\}, \\
R_{11} & :=\left\{\left(l_{1}, l_{2}\right) \in R: l_{2}<l_{1}<l_{1}+n_{1}<l_{2}+n_{2}\right\} .
\end{aligned}
$$

Then $R=\bigcup_{i=1}^{11} R_{i}$ and $R_{i} \cap R_{j}=\emptyset$ for $i \neq j$.

Put $U_{N, i}(k, \lambda, \mu)=\sum_{r=1}^{11} U_{N, i}^{(r)}(k, \lambda, \mu), i=1,2,3,4$, where $U_{N, i}^{(r)}(k, \lambda, \mu)$ has the same form as $U_{N, i}(k, \lambda, \mu)$ with $\sum_{R}$ replaced by $\sum_{R_{r}}$. By symmetry of pairs of indices $\left(l_{1}, n_{1}\right)$ and $\left(l_{2}, n_{2}\right)$, we have for $i=1,2,3$,

$$
U_{N, i}^{(r)}(k, \lambda, \mu)=U_{N, i}^{(r+6)}(k, \lambda, \mu), \quad r=1, \ldots, 5 .
$$

LEMMA 7.1. If $b_{n}$ is a sequence of complex numbers, then

$$
\sum_{l_{1}=1}^{N-n_{1}} \sum_{l_{2}=1}^{N-n_{2}} b_{l_{2}-l_{1}}=\sum_{s=-N}^{N} b_{s} V\left(s, n_{1}, n_{2}\right)
$$

where

$$
V\left(s, n_{1}, n_{2}\right)= \begin{cases}0 & \text { if }-\left(N-n_{1}\right) \geq s, \\ N-n_{1}-s & \text { if }-\left(N-n_{1}\right)<s \leq \min \left(0, n_{1}-n_{2}\right), \\ N-\max \left(n_{1}, n_{2}\right) & \text { if } \min \left(0, n_{1}-n_{2}\right) \leq s \\ & \text { if } \max \left(0, n_{1}-n_{2}\right) \leq s<N-n_{2}, \\ N-n_{2}-s & \text { if } N-n_{2} \leq s .\end{cases}
$$

Proof. See [7], p. 95. 
LEMMA 7.2. Let $a_{s}, b_{s}, c_{s}$ be sequences of nonnegative numbers such that $\sum_{s=0}^{\infty} a_{s}<\infty, \sum_{s=0}^{\infty} b_{s}<\infty$ and $\sum_{s=0}^{\infty} c_{s}<\infty$. Then for $i=2,3,4,6$,

$$
Q_{i}=\frac{1}{N^{2}} \sum_{n_{1}, n_{2}=1}^{N-1} \sum_{\left(l_{1}, l_{2}\right) \in R_{i}} a_{l_{1}} b_{l_{2}-l_{1}} c_{l_{2}-l_{1}+n_{2}-n_{1}}=O\left(\frac{1}{N}\right) \sum_{s=0}^{\infty} a_{s}
$$

and

$$
Q_{5}=\frac{1}{N^{2}} \sum_{n_{1}, n_{2}=1}^{N-1} \sum_{\left(l_{1}, l_{2}\right) \in R_{5}} a_{l_{1}} b_{l_{2}-l_{1}} c_{l_{1}-l_{2}+n_{1}-n_{2}}=O\left(\frac{1}{N}\right) \sum_{s=0}^{\infty} a_{s} .
$$

Proof. We give the proof for $i=2$; the other cases can be treated in the same way.

$$
\begin{aligned}
Q_{2}= & \frac{1}{N^{2}} \sum_{n_{1}, n_{2}=1}^{N-1} 1_{\left\{n_{1} \geq n_{2}\right\}} \sum_{\left(l_{1}, l_{2}\right) \in R_{2}} a_{l_{1}} b_{l_{2}}-l_{1} c_{l_{2}-l_{1}+n_{2}-n_{1}} \\
& +\frac{1}{N^{2}} \sum_{n_{1}, n_{2}=1}^{N-1} 1_{\left\{n_{1}<n_{2}\right\}} \sum_{\left(l_{1}, l_{2}\right) \in R_{2}} a_{l_{1}} b_{l_{2}-l_{1}} c_{l_{2}-l_{1}+n_{2}-n_{1}} \\
= & Q_{21}+Q_{22} .
\end{aligned}
$$

We have

$$
Q_{21}=\frac{1}{N^{2}} \sum_{n_{1}, n_{2}=1}^{N-1} 1_{\left\{n_{1} \geq n_{2}\right\}} \sum_{l_{1}=1}^{N-n_{1}} \sum_{s=1+n_{1}-n_{2}}^{N-n_{2}-l_{1}} a_{l_{1}} b_{s} c_{s+n_{2}-n_{1}},
$$

by putting $s=l_{2}-l_{1}$ and observing that $l_{2}-l_{1}>n_{1}-n_{2}$ in $R_{2}$. Hence

$$
\begin{aligned}
Q_{21} & =\frac{1}{N^{2}} \sum_{n_{2}=1}^{N-1} \sum_{p=0}^{N-n_{2}-1} \sum_{l_{1}=1}^{N-p-n_{2}} \sum_{r=1}^{N-n_{1}-p-l_{1}} a_{l_{1}} b_{r+p} c_{r} \\
& \leq \frac{1}{N^{2}} \sum_{n_{2}=1}^{N-1} \sum_{p=0}^{N-2} \sum_{l_{1}=1}^{N-1} \sum_{r=1}^{N-2} a_{l_{1}} b_{r+p} c_{r} \\
& \leq \frac{1}{N}\left(\sum_{n=0}^{\infty} a_{n}\right)\left(\sum_{p=0}^{\infty} b_{p}\right)\left(\sum_{r=0}^{\infty} c_{r}\right)=O\left(\frac{1}{N}\right) \sum_{s=0}^{\infty} a_{s} .
\end{aligned}
$$

We treat $Q_{22}$ in the same way and we obtain the result.

LEMMA 7.3. Let $a_{n}$ and $b_{n}$ be sequences of complex numbers such that $\sum_{n=0}^{\infty}\left|a_{n}\right|<\infty$ and $\sum_{n=0}^{\infty}\left|b_{n}\right|<\infty$ and $d$ a complex number such that $|d| \leq 1$. Put

$$
P_{i}=\frac{1}{N^{2}} \sum_{n_{1}, n_{2}=1}^{N-1} \sum_{l_{1}, l_{2} \in R_{i}} a_{l_{2}-l_{1}} d^{n_{1}-\left(l_{2}-l_{1}\right)} b_{l_{2}-l_{1}+n_{2}-n_{1}} \quad \text { for } i=2,3,4,6,
$$




$$
P_{5}=\frac{1}{N^{2}} \sum_{n_{1}, n_{2}=1}^{N-1} \sum_{l_{1}, l_{2} \in R_{5}} a_{l_{2}-l_{1}} d^{n_{2}} b_{l_{1}-l_{2}+n_{1}-n_{2}} .
$$

Then as $N \rightarrow \infty$, we have

$$
\begin{aligned}
& P_{2}=D_{N}(d)\left(\sum_{r=1}^{\infty} a_{r}\right)\left(\sum_{s=1}^{\infty} b_{s}\right)+O\left(\frac{1}{N}\right), \\
& P_{3}=D_{N}(d) a_{0}\left(\sum_{s=1}^{\infty} b_{s}\right)+O\left(\frac{1}{N}\right), \\
& P_{4}=D_{N}(d) b_{0}\left(\sum_{s=1}^{\infty} a_{s}\right)+O\left(\frac{1}{N}\right), \\
& P_{5}=D_{N}(d)\left(\sum_{r=1}^{\infty} a_{r}\right)\left(\sum_{s=1}^{\infty} b_{s}\right)+O\left(\frac{1}{N}\right), \\
& P_{6}=D_{N}(d) a_{0} b_{0}+O\left(\frac{1}{N}\right)
\end{aligned}
$$

where

$$
D_{N}(d)=\frac{1}{N} \sum_{n=1}^{N}\left(1-\frac{n}{N}\right) d^{n} .
$$

Proof. See [5], p. 181.

Lemma 7.4. Let

$$
P_{N}(\lambda)=\frac{1}{N} \sum_{n=2}^{N}\left(1-\frac{n}{N}\right) \phi(\lambda)^{n} .
$$

Then

(i)

$$
\begin{array}{ll}
\left|P_{N}(\lambda)\right| \leq 1 & \text { for all } \lambda \in \mathbb{R}, \\
\left|P_{N}(\lambda)\right| \leq \frac{\beta}{N \lambda^{2}}+\frac{4 \beta^{2}}{N^{2} \lambda^{2}} & \text { for all } \lambda \neq 0,
\end{array}
$$

(ii)

(iii)

$$
\left|P_{N}(\lambda)\right| \leq \frac{1}{N}+\frac{\beta}{N|\lambda|}+\frac{4 \beta^{2}}{N^{2} \lambda^{2}} \quad \text { for all } \lambda \neq 0 .
$$

Proof. (i) We have

$$
\left|P_{N}(\lambda)\right| \leq \frac{1}{N} \sum_{n=2}^{N}\left(1-\frac{n}{N}\right) \leq 1 .
$$

(ii) If $\lambda \neq 0$, then

$$
P_{N}(\lambda)=\frac{1}{N}\left\{\frac{\phi(\lambda)^{2}}{1-\phi(\lambda)}-\frac{\phi(\lambda)^{2}}{N[1-\phi(\lambda)]^{2}}\left(2-\phi(\lambda)-\phi(\lambda)^{N-1}\right)\right\},
$$

which gives the result. 
(iii) We write

$$
\frac{\phi(\lambda)^{2}}{1-\phi(\lambda)}=\phi(\lambda)+\frac{\phi(\lambda)}{1-\phi(\lambda)} .
$$

LEMMA 7.5. Assuming that $H$ is a bounded function with a bounded derivative, let

$S_{N}(u, a)=\int_{-\infty}^{\infty} W_{N}(u-v) P_{N}(v) \exp (-i v a) d v-\frac{2 \pi}{N} W_{N}(u) \sum_{n=2}^{N}\left(1-\frac{n}{N}\right) f_{n}(a)$.

Then

$$
S_{N}(u, a)=O\left(M_{N} / N\right) .
$$

Proof. As $H$ is a bounded function with a bounded derivative, we have $|H(u)| \leq A_{1}$ and $|H(u-v)-H(u)| \leq A_{2}|u|$. For $n \geq 2, f_{n}(u)$ and $\phi(\lambda)^{n}$ belong to $L^{1} \cap L^{2}$, therefore $\int_{-\infty}^{\infty} \phi(\lambda)^{n} \exp (-i \lambda a) d \lambda=2 \pi f_{n}(a)$ a.s. Thus

$$
\int_{-\infty}^{\infty} P_{N}(\lambda) \exp (-i \lambda a) d \lambda=\frac{2 \pi}{N} \sum_{n=2}^{N}\left(1-\frac{n}{N}\right) f_{n}(a) \quad \text { a.s. }
$$

and

$$
\begin{aligned}
\left|S_{N}(u, a)\right| & =\left|\int_{-\infty}^{\infty}\left(W_{N}(u-v)-W_{N}(u)\right) P_{N}(v) d v\right| \\
& \leq \int_{-\infty}^{\infty}\left|H\left(M_{N} u-v\right)-H\left(M_{N} u\right)\right|\left|P_{N}\left(\frac{v}{M_{N}}\right)\right| d v \\
& \leq I_{1}+I_{2}+I_{3}
\end{aligned}
$$

where

$$
\begin{aligned}
& I_{1} \leq A_{2} \int_{|v| \leq M_{N} / N}|v| d v=O\left(\frac{M_{N}^{2}}{N^{2}}\right) \\
& I_{2} \leq A_{2} \beta \int_{M_{N} / N<|v|<1}\left(\frac{|v|}{\beta N}+\frac{M_{N}}{N}+\frac{4 M_{N}^{2}}{N^{2}|v|}\right) d v=O\left(\frac{M_{N}}{N}\right), \\
& I_{3} \leq 2 A_{1} \beta^{2} \int_{|v| \geq 1}\left(\frac{1}{v^{2}}+\frac{4}{N v^{2}}\right) d v=O\left(\frac{1}{N}\right) .
\end{aligned}
$$

LEMMA 7.6. Let $h$ and $l$ belong to $L^{1}[0, \infty[$ and suppose $u h(u)$ and $u l(u)$ belong to $L^{1}[0, \infty[$. Then

$$
\sum_{n=1}^{\infty}\left(c_{n} d_{n}\right)^{1 / 2} \leq \beta\left(\int_{0}^{\infty} \int_{0}^{\infty} h(u) l(v) d u d v\right)^{1 / 2}
$$


(ii) $\sum_{n=1}^{\infty} n\left(c_{n} d_{n}\right)^{1 / 2} \leq\left(\int_{0}^{\infty} \int_{0}^{\infty}\left(\beta^{2} u+\beta\right)\left(\beta^{2} v+\beta\right) h(u) l(v) d u d v\right)^{1 / 2}$

where $c_{n}=\int_{0}^{\infty} h(u) f_{n}(u) d u$ and $d_{n}=\int_{0}^{\infty} l(u) f_{n}(u) d u$.

Pro of of Proposition 5.3. We have

$$
U_{N, 1}(k, \lambda, \mu)-E\left\{J_{k}(N, \lambda)\right\} \overline{E\left\{J_{k}(N, \mu)\right\}}=W_{N, 1}^{\prime}(k, \lambda, \mu)+W_{N, 1}^{\prime \prime}(k, \lambda, \mu)
$$

where

$$
\begin{aligned}
& W_{N, 1}^{\prime}(k, \lambda, \mu)=2 U_{N, 1}^{(1)}(k, \lambda, \mu)-E\left\{J_{k}(N, \lambda)\right\} \overline{E\left\{J_{k}(N, \mu)\right\}}, \\
& W_{N, 1}^{\prime \prime}(k, \lambda, \mu)=2 \sum_{r=2}^{5} U_{N, 1}^{(r)}(k, \lambda, \mu)+U_{N, 1}^{(6)}(k, \lambda, \mu) .
\end{aligned}
$$

Assumption $\left(\mathcal{H}_{1}\right)$ and Theorem 5.1 ensure that

$$
E\left\{J_{k}(N, \lambda)\right\}=\frac{1}{2 \pi \beta N} \sum_{n=1}^{N-1}(N-n) e_{n}^{k}(\lambda)+O\left(\frac{1}{N}\right) .
$$

As $\left|e_{n}^{k}(\lambda)\right| \leq 2 \int_{-\infty}^{\infty}\left|B_{k}(u)\right| f_{n}(u) d u=2 d_{n}^{k}$ with $\sum_{n \geq 1} d_{n}^{k}<\infty$ and $\sum_{n \geq 1} n d_{n}^{k}<\infty$, we have

$$
\begin{aligned}
E\left\{J_{k}(N, \lambda)\right\} \overline{E\left\{J_{k}(N, \mu)\right\}} & \\
& =\frac{1}{(2 \pi \beta N)^{2}} \sum_{n_{1}, n_{2}=1}^{N-1}\left(N-n_{1}\right)\left(N-n_{2}\right) e_{n_{1}}^{k} \overline{e_{n_{2}}^{k}}+O\left(\frac{1}{N}\right)
\end{aligned}
$$

where the $O(1 / N)$ is uniform in $\lambda$ and $\mu$. For $\left(l_{1}, l_{2}\right) \in R_{1}, t_{l_{1}}, t_{l_{1}+n_{1}}-t_{l_{1}}$, $t_{l_{2}}-t_{l_{1}+n_{1}}, t_{l_{2}+n_{2}}-t_{l_{2}}$ are independent, therefore

$$
\begin{aligned}
U_{N, 1}^{(1)}(k, \lambda, \mu) & (2 \pi \beta N)^{2} \\
= & \sum_{n_{1}, n_{2}=1}^{N-1} \sum_{\left(l_{1}, l_{2}\right) \in R_{1}} \int_{0}^{\infty} \int_{0}^{\infty} \int_{0}^{\infty} \int_{0}^{\infty} B\left(t_{1}, u_{1}\right) B\left(t_{2}+u_{1}+t_{1}, u_{2}\right) \\
& \times\left\{\exp \left(i\left(\lambda-\frac{2 \pi}{T} k\right) u_{1}\right)+\exp \left(-i \lambda u_{1}\right)\right\} \\
& \times\left\{\exp \left(-i\left(\mu-\frac{2 \pi}{T} k\right) u_{2}\right)+\exp \left(i \mu u_{2}\right)\right\} \\
& \times \exp \left\{i \frac{2 \pi}{T} k\left(t_{2}+u_{1}\right)\right\} \\
& \times f_{l_{1}}\left(t_{1}\right) f_{n_{1}}\left(u_{1}\right) f_{l_{2}-l_{1}-n_{1}}\left(t_{2}\right) f_{n_{2}}\left(u_{2}\right) d t_{1} d t_{2} d u_{1} d u_{2}
\end{aligned}
$$




$$
\begin{aligned}
= & \sum_{n_{1}, n_{2}=1}^{N-1} \sum_{\left(l_{1}, l_{2}\right) \in R_{1}} \sum_{j_{1}+j_{2}=0} \int_{0}^{\infty} \int_{0}^{\infty}\left\{\exp \left(i\left(\lambda-\frac{2 \pi}{T} k\right) u_{1}\right)\right. \\
& \left.+\exp \left(-i \lambda u_{1}\right)\right\} \times\left\{\exp \left(-i\left(\mu-\frac{2 \pi}{T} k\right) u_{2}\right)+\exp \left(i \mu u_{2}\right)\right\} \\
& \times \phi\left(\frac{2 \pi}{T}\left(j_{2}+k\right)\right)^{l_{2}-l_{1}-n_{1}} B_{j_{1}}\left(u_{1}\right) B_{j_{2}}\left(u_{2}\right) f_{n_{1}}\left(u_{1}\right) f_{n_{2}}\left(u_{2}\right) d u_{1} d u_{2} \\
& +\sum_{n_{1}, n_{2}=1}^{N-1} \sum_{j_{1}+j_{2} \neq 0} \int_{0}^{\infty} \int_{0}^{\infty} B_{j_{1}}\left(u_{1}\right) B_{j_{2}}\left(u_{2}\right) \exp \left(i \frac{2 \pi}{T}\left(j_{2}+k\right) u_{1}\right) \\
& \times\left\{\exp \left(i\left(\lambda-\frac{2 \pi}{T} k\right) u_{1}\right)+\exp \left(-i \lambda u_{1}\right)\right\} \\
& \times\left\{\exp \left(-i\left(\mu-\frac{2 \pi}{T} k\right) u_{2}\right)+\exp \left(i \mu u_{2}\right)\right\} \\
& \times \sum_{\left(l_{1}, l_{2}\right) \in R_{1}} \phi\left(\frac{2 \pi}{T}\left(j_{1}+j_{2}\right)\right)^{l_{1}} \phi\left(\frac{2 \pi}{T}\left(j_{2}+k\right)\right)^{l_{2}-l_{1}-n_{1}} \\
& \times f_{n_{1}}\left(u_{1}\right) f_{n_{2}}\left(u_{2}\right) d u_{1} d u_{2} \\
= & \left(Y_{1}+Y_{2}+Y_{3}\right)(2 \pi \beta N)^{2} .
\end{aligned}
$$

We have

$$
\begin{aligned}
\left|Y_{3}\right| \leq & \frac{1}{(\pi \beta N)^{2}} \sum_{n_{1}, n_{2}=1}^{N-1} \sum_{j_{1}+j_{2} \neq 0} \int_{0}^{\infty} \int_{0}^{\infty}\left|B_{j_{1}}\left(u_{1}\right) B_{j_{2}}\left(u_{2}\right)\right| \\
& \times \sum_{\left(l_{1}, l_{2}\right) \in R_{1}}\left|\phi\left(\frac{2 \pi}{T}\left(j_{1}+j_{2}\right)\right)\right|^{l_{1}} f_{n_{1}}\left(u_{1}\right) f_{n_{2}}\left(u_{2}\right) d u_{1} d u_{2} \\
\leq & \frac{1}{(\pi \beta N)^{2}} \sum_{n_{1}, n_{2}=1}^{N-1} \sum_{j_{1}+j_{2} \neq 0} \int_{0}^{\infty} \int_{0}^{\infty}\left|B_{j_{1}}\left(u_{1}\right) B_{j_{2}}\left(u_{2}\right)\right| \\
& \times \sum_{l_{1}=1}^{N-n_{1}} \sum_{s=n_{1}}^{N-n_{2}-l_{1}}\left|\phi\left(\frac{2 \pi}{T}\left(j_{1}+j_{2}\right)\right)\right|^{l_{1}} f_{n_{1}}\left(u_{1}\right) f_{n_{2}}\left(u_{2}\right) d u_{1} d u_{2} .
\end{aligned}
$$

Putting $s=l_{2}-l_{1}$ and observing that $l_{2}-l_{1} \geq n_{1}$ in $R_{1}$, we get

$$
\begin{aligned}
\left|Y_{3}\right| \leq \frac{1}{(\pi \beta)^{2} N} \frac{T}{2 \pi} \sum_{n_{1}, n_{2}=1}^{N-1} \sum_{j_{1}+j_{2} \neq 0} \int_{0}^{\infty} \int_{0}^{\infty} \frac{\left|B_{j_{1}}\left(u_{1}\right) B_{j_{2}}\left(u_{2}\right)\right|}{\left|j_{1}+j_{2}\right|} \\
\times f_{n_{1}}\left(u_{1}\right) f_{n_{2}}\left(u_{2}\right) d u_{1} d u_{2}=O(1 / N) .
\end{aligned}
$$


Next,

$$
\begin{aligned}
&\left.\left.\left|Y_{2}\right| \leq \frac{1}{(\pi \beta)^{2} N}\left(\frac{\beta T}{2 \pi}+1\right) \sum_{n_{1}, n_{2}=1}^{N-1} \sum_{\substack{j \neq k \\
\times f_{n_{1}}}} \int_{0}^{\infty} \int_{0}^{\infty} \mid u_{1}\right) f_{n_{2}}\left(u_{2}\right) d u_{1}\right) B_{j}\left(u_{2}\right) \mid \\
& d u_{2}=O(1 / N),
\end{aligned}
$$

therefore

$$
\begin{aligned}
W_{N, 1}^{\prime}(k, \lambda, \mu)=\frac{1}{(2 \pi \beta)^{2}} & \sum_{n_{1}, n_{2}=1}^{N-1} e_{n_{1}}^{k}(\lambda) \overline{e_{n_{2}}^{k}(\mu)} \\
\times & \frac{1}{N^{2}}\left\{2 \sum_{R_{1}} 1-\left(N-n_{1}\right)\left(N-n_{2}\right)\right\}+O\left(\frac{1}{N}\right) .
\end{aligned}
$$

On the other hand, by Lemma 7.1,

$$
\begin{aligned}
2 \sum_{R_{1}} 1 & =2 \sum_{s=n_{1}}^{N-n_{2}}\left(N-n_{2}-s\right) 1_{\left\{N-n_{1}-n_{2}-1 \geq 0\right\}} \\
& =\left(N-n_{2}-n_{1}\right)\left(N-n_{2}-n_{1}+1\right) 1_{\left\{N-n_{1}-n_{2}-1 \geq 0\right\}} .
\end{aligned}
$$

Hence

$$
\begin{aligned}
& \frac{1}{N^{2}}\left\{2 \sum_{R_{1}} 1-\left(N-n_{1}\right)\left(N-n_{2}\right)\right\} \\
&=\left\{\left(1-\frac{n_{1}+n_{2}}{N}\right)\left(1-\frac{n_{1}+n_{2}-1}{N}\right)\right. \\
&\left.-\left(1-\frac{n_{1}}{N}\right)\left(1-\frac{n_{2}}{N}\right)\right\} 1_{\left\{N-n_{1}-n_{2}-1 \geq 0\right\}} \\
&+\left(1-\frac{n_{1}}{N}\right)\left(1-\frac{n_{2}}{N}\right)\left\{1-1_{\left\{N-n_{1}-n_{2}-1 \geq 0\right\}}\right\} \\
&= A\left(n_{1}, n_{2}\right)+B\left(n_{1}, n_{2}\right) .
\end{aligned}
$$

We have

$$
\left|A\left(n_{1}, n_{2}\right)\right| \leq \frac{3}{N}\left(\frac{4}{3} n_{1}+n_{2}+1\right) \quad \text { and } \quad\left|B\left(n_{1}, n_{2}\right)\right| \leq \frac{n_{1}+1}{N},
$$

therefore

$$
\begin{aligned}
\left|W_{N, 1}^{\prime}(k, \lambda, \mu)\right| \leq & \frac{1}{(\pi \beta)^{2} N} \sum_{n_{1}, n_{2}=1}^{N-1} \int_{0}^{\infty} \int_{0}^{\infty}\left|B_{k}\left(u_{1}\right) B_{k}\left(u_{2}\right)\right| \\
& \times f_{n_{1}}\left(u_{1}\right) f_{n_{2}}\left(u_{2}\right)\left\{4 n_{1}+3 n_{2}+3+n_{1}+1\right\} d u_{1} d u_{2} \\
\leq & \frac{1}{(2 \pi \beta)^{2} N} \int_{0}^{\infty} \int_{0}^{\infty}\left|B_{k}\left(u_{1}\right) B_{k}\left(u_{2}\right)\right| \\
& \times\left\{\left(\beta^{2} u_{1}+\beta\right) \beta+\left(\beta^{2} u_{2}+\beta\right) \beta+\beta^{2}\right\} d u_{1} d u_{2}=O(1 / N)
\end{aligned}
$$

using assumption $\left(\mathcal{H}_{2}\right)$. 
Let us now evaluate $W_{N, 1}^{\prime \prime}(k, \lambda, \mu)$. For $r=2, \ldots, 6, t_{l_{1}}$ and $t_{l_{1}+n_{1}}-t_{l_{1}}$, $t_{l_{1}}$ and $t_{l_{2}+n_{2}}-t_{l_{2}}, t_{l_{1}}$ and $t_{l_{2}}-t_{l_{1}}$ are independent pairs of r.v.'s; therefore

$$
\begin{aligned}
\left|U_{N, 1}^{(r)}(k, \lambda, \mu)\right| \leq \frac{4}{(2 \pi \beta N)^{2}} \sum_{n_{1}, n_{2}=1}^{N-1} \sum_{\left(l_{1}, l_{2}\right) \in R_{r}} \sum_{\left(j_{1}, j_{2}\right) \in \mathbb{Z}^{2}}\left|\phi\left(\frac{2 \pi}{T}\left(j_{1}+j_{2}\right)\right)^{l_{1}}\right| \\
\times E\left[h_{j_{1}}\left(t_{l_{1}+n_{1}}-t_{l_{1}}\right) h_{j_{2}}\left(t_{l_{2}+n_{2}}-t_{l_{2}}\right)\right] .
\end{aligned}
$$

Let $\gamma=t_{l_{1}+n_{1}}-t_{l_{1}}$ and $\delta=t_{l_{2}+n_{2}}-t_{l_{2}}$, and choose $\gamma^{\prime} \leq \gamma$ and $\delta^{\prime} \leq \delta, \gamma^{\prime}$ and $\delta^{\prime}$ being independent for $r=2,3,4,5,6$ :

$$
\gamma \geq \begin{cases}t_{l_{2}}-t_{l_{1}}, & r=2,4, \\ t_{l_{1}+n_{1}}-t_{l_{1}}, & r=3,6, \\ \left(t_{l_{2}}-t_{l_{1}}\right)+\left(t_{l_{1}+n_{1}}-t_{l_{2}+n_{2}}\right), & r=5,\end{cases}
$$

and

$$
\delta \geq \begin{cases}t_{l_{2}+n_{2}}-t_{l_{1}+n_{1}}, & r=3, \\ t_{l_{2}+n_{2}}-t_{l_{2}}, & r=2,4,5, \\ 0, & r=6 .\end{cases}
$$

Since $h_{j}(u)$ is decreasing on $[0, \infty[$, we have

$$
E\left\{h_{j_{1}}(\gamma) h_{j_{2}}(\delta)\right\} \leq E\left\{h_{j_{1}}\left(\gamma^{\prime}\right) h_{j_{2}}\left(\delta^{\prime}\right)\right\} \leq E\left\{h_{j_{1}}\left(\gamma^{\prime}\right)\right\} E\left\{h_{j_{2}}\left(\delta^{\prime}\right)\right\} .
$$

Notice that the probability density of $\left(t_{l_{2}}-t_{l_{1}}\right)+\left(t_{l_{1}+n_{1}}-t_{l_{2}+n_{2}}\right)$ is $f_{n_{2}-n_{1}}$, so for $r=2, \ldots, 6$,

$$
\begin{aligned}
& \left|U_{N, 1}^{(r)}(k, \lambda, \mu)\right| \leq G_{N, 1}^{(r)} \\
& =\frac{1}{(\pi \beta N)^{2}} \sum_{n_{1}, n_{2}=1}^{N-1} \sum_{\left(l_{1}, l_{2}\right) \in R_{r}} \sum_{\left(j_{1}, j_{2}\right) \in \mathbb{Z}^{2}}\left|\phi\left(\frac{2 \pi}{T}\left(j_{1}+j_{2}\right)^{l_{1}}\right)^{l_{1}}\right| g^{(r)}\left(l_{1}, l_{2}, n_{1}, n_{2}\right),
\end{aligned}
$$

where

$$
g^{(r)}\left(l_{1}, l_{2}, n_{1}, n_{2}\right)= \begin{cases}a_{j_{1}}\left(l_{2}-l_{1}\right) a_{j_{2}}\left(n_{2}\right), & r=2, \\ a_{j_{1}}\left(n_{1}\right) a_{j_{2}}\left(n_{2}-n_{1}+l_{2}-l_{1}\right), & r=3, \\ a_{j_{1}}\left(l_{2}-l_{1}\right) a_{j_{2}}\left(n_{2}\right), & r=4, \\ a_{j_{1}}\left(n_{1}-n_{2}\right) a_{j_{2}}\left(n_{2}\right), & r=5, \\ a_{j_{1}}\left(n_{1}\right), & r=6,\end{cases}
$$

with $a_{j}(n)=\int_{0}^{\infty} h_{j}(u) f_{n}(u) d u$. $R_{5}$ being characterized by $l_{2}-l_{1}>0$ and $l_{2}-l_{1}<n_{1}-n_{2}$, putting $s=l_{2}-l_{1}$, we have

$$
\begin{aligned}
G_{N, 1}^{(5)}= & \frac{1}{(\pi \beta N)^{2}} \sum_{n_{1}, n_{2}=1}^{N-1} \sum_{j_{1}+j_{2} \neq 0} \sum_{l_{1}=1}^{N-n_{1}} \sum_{s=1}^{n_{1}-n_{2}-1} a_{j_{1}}\left(n_{1}-n_{2}\right) a_{j_{2}}\left(n_{2}\right) \\
& \times\left|\phi\left(\frac{2 \pi}{T}\left(j_{1}+j_{2}\right)\right)^{l_{1}}\right| 1_{\left\{n_{1}-n_{2}-2 \geq 0\right\}}
\end{aligned}
$$




$$
\begin{aligned}
& +\frac{1}{(\pi \beta N)^{2}} \sum_{n_{1}, n_{2}=1}^{N-1} \sum_{j_{1}+j_{2}=0} \sum_{s=1}^{n_{1}-n_{2}-1}\left(N-n_{1}\right) \\
& \times a_{j_{1}}\left(n_{1}-n_{2}\right) a_{j_{2}}\left(n_{2}\right) 1_{\left\{n_{1}-n_{2}-2 \geq 0\right\}} \\
\leq & \frac{T}{2 \pi(\pi \beta)^{2} N} \sum_{n_{2}=1}^{N-1} \sum_{p=2}^{N-1-n_{2}} \sum_{j_{1}+j_{2} \neq 0} \frac{a_{j_{1}}(p) a_{j_{2}}\left(n_{2}\right)}{\left|j_{1}+j_{2}\right|} \\
& +\frac{1}{(\pi \beta)^{2} N} \sum_{n_{2}=1}^{N-1} \sum_{p=2}^{N-n_{2}-1} \sum_{j_{1}+j_{2}=0}(p-1) a_{j_{1}}(p) a_{j_{2}}\left(n_{2}\right) \\
\leq & \frac{T}{2 \pi^{3} N} \sum_{j_{1}+j_{2} \neq 0} \int_{0}^{\infty} \int_{0}^{\infty} \frac{h_{j_{1}}\left(u_{1}\right) h_{j_{2}}\left(u_{2}\right)}{\left|j_{1}+j_{2}\right|} d u_{1} d u_{2} \\
& +\frac{1}{(\pi \beta)^{2} N} \sum_{j_{1}+j_{2}=0} \int_{0}^{\infty} \int_{0}^{\infty}\left(\beta^{2} u_{1}+2 \beta\right) h_{j_{1}}\left(u_{1}\right) h_{j_{2}}\left(u_{2}\right) d u_{1} d u_{2} \\
= & O\left(\frac{1}{N}\right) .
\end{aligned}
$$

In the same way $G_{N, 1}^{(r)}=O(1 / N)$ for $r=2,3,4,6$, therefore $W_{N, 1}^{\prime \prime}(k, \lambda, \mu)=$ $O(1 / N)$ uniformly in $\lambda$ and $\mu$.

To show that $U_{N, 3}(k, \lambda, \mu)=O(1 / N)$ and $U_{N, 4}(k, \lambda, \mu)=O(1 / N)$, we use the method employed to evaluate $W_{N, 1}^{\prime \prime}(k, \lambda, \mu)$.

Proof of Proposition 5.4. As for $W_{N, 1}^{\prime \prime}(k, \lambda, \mu)$, we have

$$
U_{N, 2}^{(1)}(k, \lambda, \mu)=O(1 / N)
$$

uniformly in $\lambda$ and $\mu$.

For $r=2,3,4,6$, writing

$$
t_{l_{2}}=\left(t_{l_{2}}-t_{l_{1}}\right)+t_{l_{1}}, \quad t_{l_{1}+n_{1}}-t_{l_{1}}=\left(t_{l_{1}+n_{1}}-t_{l_{2}}\right)+\left(t_{l_{2}}-t_{l_{1}}\right)
$$

and

$$
t_{l_{2}+n_{2}}-t_{l_{2}}=\left(t_{l_{2}+n_{2}}-t_{l_{1}+n_{1}}\right)+\left(t_{l_{1}+n_{1}}-t_{l_{2}}\right),
$$

we have

$$
\begin{aligned}
U_{N, 2}^{(r)}(k, \lambda, \mu)= & \Omega(\lambda, \mu)+\Omega\left(\lambda,-\mu+\frac{2 \pi}{T} k\right) \\
& +\Omega\left(-\lambda+\frac{2 \pi}{T} k, \mu\right) \\
& +\Omega\left(-\lambda+\frac{2 \pi}{T} k,-\mu+\frac{2 \pi}{T} k\right)
\end{aligned}
$$


where

$$
\begin{aligned}
\Omega(\lambda, \mu)= & \frac{1}{(2 \pi \beta N)^{2}} \sum_{n_{1}, n_{2}=1}^{N-1} \sum_{\left(l_{1}, l_{2}\right) \in R_{r}} E\left(B_{\left.j_{1}, j_{2}\right) \in \mathbb{Z}^{2}}\left(t_{l_{2}}-t_{l_{1}}\right) B_{j_{2}}\left(t_{l_{2}+n_{2}}-t_{l_{1}+n_{1}}\right)\right. \\
& \times \exp \left(i \frac{2 \pi}{T} j_{2}\left(t_{l_{1}+n_{2}}-t_{l_{2}}\right)\right) \\
& \times \exp \left(i \frac{2 \pi}{T}\left(j_{1}+j_{2}\right) t_{l_{1}}\right) \\
& \times \exp \left(i \frac{2 \pi}{T}\left(j_{2}+k\right)\left(t_{l_{2}}-t_{l_{1}}\right)\right) \\
& \times \exp \left(i\left(\lambda-\frac{2 \pi}{T} k\right)\left(t_{l_{2}}-t_{l_{1}}\right)\right) \exp \left(i(\lambda-\mu)\left(t_{l_{1}+n_{1}}-t_{l_{2}}\right)\right) \\
& \times \exp \left(-i\left(\mu-\frac{2 \pi}{T} k\right)\left(t_{l_{2}+n_{2}}-t_{l_{1}+n_{1}}\right)\right) \\
& \left.\times \exp \left(i \frac{2 \pi}{T}\left(j_{1}+j_{2}\right) t_{l_{1}}\right)\right) .
\end{aligned}
$$

Since for $r=2,3,4,6, t_{l_{2}+n_{2}}-t_{l_{1}+n_{1}}$ and $t_{l_{1}+n_{1}}-t_{l_{2}}, t_{l_{2}}-t_{l_{1}}$ are independent,

$$
\begin{aligned}
\Omega(\lambda, \mu)= & \frac{1}{(2 \pi \beta N)^{2}} \sum_{n_{1}, n_{2}=1}^{N-1} \sum_{\left(l_{1}, l_{2}\right) \in R_{r}} \sum_{\left(j_{1}, j_{2}\right) \in \mathbb{Z}^{2}} \phi\left(\frac{2 \pi}{T}\left(j_{1}+j_{2}\right)\right)^{l_{1}} \\
& \times z_{j_{1}}\left(l_{2}-l_{1}, \lambda+\frac{2 \pi}{T} j_{2}\right) \phi\left(\lambda-\mu+\frac{2 \pi}{T} j_{2}\right)^{n_{1}-\left(l_{2}-l_{1}\right)} \\
& \times z_{j_{2}}\left(n_{2}-n_{1}+l_{2}-l_{1},-\mu+\frac{2 \pi}{T} k\right)
\end{aligned}
$$

where $z_{j}(n, \lambda)=\int_{0}^{\infty} B_{j}(u) f_{n}(u) \exp (i \lambda u) d u$ for $n \geq 1$ and $z_{j}(0, \lambda)=B_{j}(0)$. Hence

$$
\begin{aligned}
\Omega(\lambda, \mu)= & \frac{1}{(2 \pi \beta N)^{2}} \sum_{n_{1}, n_{2}=1}^{N-1} \sum_{\left(l_{1}, l_{2}\right) \in R_{r}} \sum_{j_{1}+j_{2}=0} z_{j_{1}}\left(l_{2}-l_{1}, \lambda-\frac{2 \pi}{T} j_{1}\right) \\
& \times \phi\left(\lambda-\mu-\frac{2 \pi}{T} j_{1}\right)^{n_{1}-\left(l_{2}-l_{1}\right)} z_{j_{2}}\left(n_{2}-n_{1}+l_{2}-l_{1},-\mu+\frac{2 \pi}{T} k\right) \\
& +Q(\lambda, \mu)
\end{aligned}
$$


where

$|Q(\lambda, \mu)|$

$$
\leq \frac{1}{(2 \pi \beta N)^{2}} \sum_{n_{1}, n_{2}=1}^{N-1} \sum_{j_{1}+j_{2} \neq 0} \sum_{\left(l_{1}, l_{2}\right) \in R_{r}} a_{l_{1}} b_{j_{1}}\left(l_{2}-l_{1}\right) b_{j_{2}}\left(n_{2}-n_{1}+l_{2}-l_{1}\right)
$$

with $a_{l}=\left|\phi\left(\frac{2 \pi}{T}\left(j_{1}+j_{2}\right)\right)^{l}\right|, b_{j}(l)=\int_{0}^{\infty}\left|B_{j}(u)\right| f_{l}(u) d u, l \geq 1$, and $b_{j}(0)=$ $\left|B_{j}(0)\right|$. Lemma 7.2 implies that

$$
\begin{aligned}
|Q(\lambda, \mu)| & \leq \frac{1}{(2 \pi \beta)^{2} N} \sum_{j_{1}+j_{2} \neq 0} \sum_{l=1}^{\infty} \sum_{p=1}^{\infty} \sum_{s=1}^{\infty} a_{l} b_{j_{1}}(s) b_{j_{2}}(p) \\
& \leq \frac{1}{(2 \pi \beta)^{2} N} \sum_{j_{1}+j_{2} \neq 0} \int_{0}^{\infty} \int_{0}^{\infty} \frac{\left|B_{j_{1}}\left(u_{1}\right) B_{j_{2}}\left(u_{2}\right)\right|}{\left|j_{1}+j_{2}\right|} d u_{1} d u_{2}=O\left(\frac{1}{N}\right)
\end{aligned}
$$

where the $O(1 / N)$ term is uniform in $\lambda$ and $\mu$.

To evaluate $U_{N, 2}^{(5)}(k, \lambda, \mu)$, we write $t_{l_{2}+n_{2}}-t_{l_{1}+n_{1}}=-\left(t_{l_{1}+n_{1}}-t_{l_{2}+n_{2}}\right)$ and $t_{l_{1}+n_{1}}-t_{l_{1}}=\left(t_{l_{1}+n_{1}}-t_{l_{2}+n_{2}}\right)+\left(t_{l_{2}+n_{2}}-t_{l_{2}}\right)+\left(t_{l_{2}}-t_{l_{1}}\right)$ and we use the formula $B_{j}(-u)=\exp \left(-i \frac{2 \pi}{T} j u\right) B_{j}(u)$; then the same method as above gives us

$$
\begin{aligned}
U_{N, 1}^{(5)}(k, \lambda, \mu)= & \Omega^{\prime}(\lambda, \mu)+\Omega^{\prime}\left(\lambda,-\mu+\frac{2 \pi}{T} k\right) \\
& +\Omega^{\prime}\left(-\lambda+\frac{2 \pi}{T} k, \mu\right)+\Omega^{\prime}\left(-\lambda+\frac{2 \pi}{T} k,-\mu+\frac{2 \pi}{T} k\right)
\end{aligned}
$$

where

$$
\begin{aligned}
\Omega^{\prime}(\lambda, \mu)= & \frac{1}{(2 \pi \beta N)^{2}} \sum_{n_{1}, n_{2}=1}^{N-1} \sum_{\left(l_{1}, l_{2}\right) \in R_{r}} \sum_{j_{1}+j_{2}=0} z_{j_{1}}\left(l_{2}-l_{1}, \lambda-\frac{2 \pi}{T} j_{1}\right) \\
& \quad \times \phi\left(\lambda-\mu-\frac{2 \pi}{T} j_{1}\right)^{n_{2}} z_{j_{2}}\left(n_{1}-n_{2}+l_{1}-l_{2},-\mu+\frac{2 \pi}{T} k\right) \\
& +O\left(\frac{1}{N}\right) .
\end{aligned}
$$

Put $\bar{D}_{N}(\lambda)=D_{N}(\phi(\lambda))$. Then Lemma 7.3 gives us

$$
\begin{aligned}
U_{N, 2}(k, \lambda, \mu)= & 2 \sum_{r=1}^{5} U_{N, 2}^{(r)}(k, \lambda, \mu)+U_{N, 2}^{(6)} \\
= & S(\lambda, \mu)+S\left(-\lambda+\frac{2 \pi}{T} k\right)+S\left(\lambda,-\mu+\frac{2 \pi}{T} k\right) \\
& +S\left(-\lambda+\frac{2 \pi}{T} k,-\mu+\frac{2 \pi}{T} k\right)+O\left(\frac{1}{N}\right)
\end{aligned}
$$


where

$$
\begin{aligned}
S(\lambda, \mu)=\frac{1}{(2 \pi \beta)^{2}} \sum_{j \in \mathbb{Z}} \bar{D}_{N}\left(\lambda-\mu-\frac{2 \pi}{T} j\right)\left(2 Z_{j}\left(\lambda-\frac{2 \pi}{T} j\right) \overline{Z_{j}\left(\mu-\frac{2 \pi}{T} k\right)}\right. \\
+2 B_{j}(0) \overline{Z_{j}\left(\mu-\frac{2 \pi}{T} k\right)}+2 Z_{j}\left(\lambda-\frac{2 \pi}{T} j\right) \overline{B_{j}(0)} \\
\left.+B_{j}(0) \overline{B_{j}(0)}+2 Z_{j}\left(\lambda-\frac{2 \pi}{T} j\right) \overline{Z_{j}\left(-\lambda+\frac{2 \pi}{T} k\right)}\right)
\end{aligned}
$$

and $Z_{j}(\lambda)=\sum_{n=1}^{\infty} z_{j}(n, \lambda)=\int_{0}^{\infty} B_{j}(u) \exp (i \lambda u) d u$. We have

$$
\int_{-\infty}^{\infty} \int_{-\infty}^{\infty} W_{N}(u-\lambda) W_{N}(u-\mu) S(\lambda, \mu) d \lambda d \mu=\sum_{i=1}^{5} T_{i} .
$$

For example, writing $\bar{D}_{N}(\lambda)=\frac{N-1}{N^{2}} \phi(\lambda)+P_{N}(\lambda)$, we have $T_{1}=T_{11}+T_{12}$ where

$$
\begin{aligned}
\left|T_{12}\right| \leq & \frac{A_{4}}{N} \int_{-\infty}^{\infty} \int_{-\infty}^{\infty}\left|W_{N}(u-\lambda) W_{N}(u-\mu)\right| d \lambda d \mu \\
& \times \sum_{j \in \mathbb{Z}} \int_{0}^{\infty} \int_{0}^{\infty}\left|B_{j}(s) B_{j}(t)\right| d s d t=O\left(\frac{1}{N}\right)
\end{aligned}
$$

by (iii) of assumption $\left(\mathcal{H}_{2}\right)$, and

$$
\begin{aligned}
T_{11}= & \frac{1}{(2 \pi \beta)^{2}} \int_{-\infty}^{\infty} \int_{-\infty}^{\infty} \sum_{j \in \mathbb{Z}} W_{N}(u-\lambda) W_{N}(u-\mu) P_{N}\left(\lambda-\mu-\frac{2 \pi}{T} j\right) \\
& \times\left(\int_{0}^{\infty} \int_{0}^{\infty} B_{j}(s) B_{-j}(t) \exp \left(i\left(\lambda-\frac{2 \pi}{T} j\right) s\right)\right. \\
& \left.\times \exp \left(-i\left(\mu-\frac{2 \pi}{T} k\right) t\right) d s d t\right) d \lambda d \mu \\
= & \frac{1}{(2 \pi \beta)^{2}} \int_{-\infty}^{\infty} \int_{0}^{\infty} \int_{0}^{\infty} W_{N}(u-\lambda) \sum_{j \in \mathbb{Z}} B_{j}(s) B_{-j}(t) \\
& \times \exp \left(i\left(\lambda-\frac{2 \pi}{T} j\right) s\right) \exp \left(-i\left(\lambda-\frac{2 \pi}{T} j-\frac{2 \pi}{T} k\right) t\right) d \lambda d s d t \\
& \times\left(\int_{-\infty}^{\infty} W_{N}\left(u-\lambda+\frac{2 \pi}{T} j+v\right) P_{N}(v) \exp (i v t) d v\right) .
\end{aligned}
$$


Lemma 7.5 gives us

$$
\begin{aligned}
T_{11}= & \frac{1}{2 \pi \beta^{2}} \int_{-\infty}^{\infty} \int_{0}^{\infty} \int_{0}^{\infty} W_{N}(u-\lambda) \sum_{j \in \mathbb{Z}} B_{j}(s) B_{-j}(t) \\
& \times \exp \left(i\left(\lambda-\frac{2 \pi}{T} j\right) s\right) \exp \left(-i\left(\lambda-\frac{2 \pi}{T} j-\frac{2 \pi}{T} k\right) t\right) \\
& \times\left(\frac{2 \pi}{N} W_{N}\left(u-\lambda+\frac{2 \pi}{T} j\right) \sum_{n=2}^{N}\left(1-\frac{n}{N}\right) f_{n}(-t)\right) d \lambda d s d t \\
& +O\left(\frac{M_{N}}{N}\right)
\end{aligned}
$$

and therefore $T_{11}=0+O\left(M_{N} / N\right)$.

We treat the other $T_{i}$ in the same way and we obtain the result.

Proof of Proposition 5.5. We have

$$
\operatorname{var}\left\{\Gamma_{k}(n, N, \omega)\right\}=\sum_{i=1}^{4} U_{n, N, i}(k, \omega)-\left|E\left\{\Gamma_{k}(n, N, \omega)\right\}\right|^{2}
$$

where

$$
\begin{aligned}
& U_{n, N, 1}(k, \omega)=\frac{1}{N^{2}} \sum_{\left(l_{1}, l_{2}\right) \in R^{\prime}} E\left(B\left(t_{l_{1}}, t_{l_{1}+n}-t_{l_{1}}\right) B\left(t_{l_{2}}, t_{l_{2}+n}-t_{l_{2}}\right)\right. \\
& \left.\times p\left(l_{1}, n, k, \omega\right) \overline{p\left(l_{2}, n, k, \omega\right)}\right), \\
& U_{n, N, 2}(k, \omega)=\frac{1}{N^{2}} \sum_{\left(l_{1}, l_{2}\right) \in R^{\prime}} E\left(B\left(t_{l_{1}}, t_{l_{2}}-t_{l_{1}}\right) B\left(t_{l_{1}+n}, t_{l_{2}+n}-t_{l_{1}+n}\right)\right. \\
& \left.\times p\left(l_{1}, n, k, \omega\right) \overline{p\left(l_{2}, n, k, \omega\right)}\right), \\
& U_{n, N, 3}(k, \omega)=\frac{1}{N^{2}} \sum_{\left(l_{1}, l_{2}\right) \in R^{\prime}} E\left(B\left(t_{l_{1}}, t_{l_{2}+n}-t_{l_{1}}\right) B\left(t_{l_{2}}, t_{l_{1}+n}-t_{l_{2}}\right)\right. \\
& \left.\times p\left(l_{1}, n, k, \omega\right) \overline{p\left(l_{2}, n, k, \omega\right)}\right), \\
& U_{n, N, 4}(k, \omega)=\frac{1}{N^{2}} \sum_{\left(l_{1}, l_{2}\right) \in R^{\prime}} E\left(K\left(t_{l_{1}}, t_{l_{1}+n}-t_{l_{1}}, t_{l_{2}+n}-t_{l_{1}}, t_{l_{2}}-t_{l_{1}}\right)\right. \\
& \left.\times p\left(l_{1}, n, k, \omega\right) \overline{p\left(l_{2}, n, k, \omega\right)}\right)
\end{aligned}
$$

with $R^{\prime}=\left\{\left(l_{1}, l_{2}\right) \in \mathbb{N}: 1 \leq l_{1}, l_{2} \leq N-n\right\}$. Put

$$
\begin{aligned}
& R_{1}^{\prime}=\left\{\left(l_{1}, l_{2}\right) \in R^{\prime}: l_{1}<l_{1}+n \leq l_{2}<l_{2}+n\right\}, \\
& R_{2}^{\prime}=\left\{\left(l_{1}, l_{2}\right) \in R^{\prime}: l_{1}<l_{2}<l_{1}+n<l_{2}+n\right\}, \\
& R_{3}^{\prime}=\left\{\left(l_{1}, l_{2}\right) \in R^{\prime}: l_{2}<l_{2}+n \leq l_{1}<l_{1}+n\right\}, \\
& R_{4}^{\prime}=\left\{\left(l_{1}, l_{2}\right) \in R^{\prime}: l_{2}<l_{1}<l_{2}+n<l_{1}+n\right\},
\end{aligned}
$$




$$
R_{5}^{\prime}=\left\{\left(l_{1}, l_{2}\right) \in R^{\prime}: l_{1}=l_{2}<l_{1}+n=l_{2}+n\right\} .
$$

Then $R^{\prime}=\bigcup_{r=1}^{5} R_{r}^{\prime}$ and $R_{i}^{\prime} \cap R_{j}^{\prime}=\emptyset$ for $i \neq j$, therefore $U_{n, N, 1}(k, \omega)=$ $\sum_{r=1}^{5} U_{n, N, 1}^{(r)}(k, \omega)$ where $U_{n, N, 1}^{(r)}(k, \omega)$ has the same form as $U_{n, N, 1}(k, \omega)$ with $\sum_{R^{\prime}}$ replaced by $\sum_{R_{r}^{\prime}}$. Moreover, by symmetry of $l_{1}$ and $l_{2}$, we have $U_{n, N, 1}^{(1)}(k, \omega)=U_{n, N, 1}^{(3)}(k, \omega)$ and $U_{n, N, 1}^{(2)}(k, \omega)=U_{n, N, 1}^{(4)}(k, \omega)$.

As in the proof of Proposition 5.3, we have $2 U_{n, N, 1}^{(1)}(k, \omega)-$ $\left|E\left\{\Gamma_{k}(n, N, \omega)\right\}\right|^{2}=O(1 / N)$, with the $O(1 / N)$ term uniform in $\omega$ and $k$. Let

$$
\begin{aligned}
\left|w_{2}\right|= & 2\left|U_{n, N, 1}^{(2)}(k, \omega)\right| \\
\leq & \frac{8}{N^{2}} \sum_{\left(l_{1}, l_{2}\right) \in R_{2}^{\prime}} \sum_{j_{1}, j_{2} \in \mathbb{Z}} E\left\{\left|B_{j_{1}}\left(t_{l_{1}+n}-t_{l_{1}}\right) B_{j_{2}}\left(t_{l_{2}+n}-t_{l_{2}}\right)\right|\right\} \\
& \times\left|\phi\left(\frac{2 \pi}{T}\left(j_{1}+j_{2}\right)\right)\right|^{l_{1}} \\
\leq & \frac{8}{N^{2}} \sum_{\left(l_{1}, l_{2}\right) \in R_{2}^{\prime}}\left(\sum_{j_{1}+j_{2} \neq 0} \frac{\left(c_{j_{1}}(n) c_{j_{2}}(n)\right)^{1 / 2}}{\left|j_{1}+j_{2}\right|}+\sum_{j \in \mathbb{Z}} c_{j}(n)\right)
\end{aligned}
$$

where $c_{j}(n)=\int_{0}^{\infty}\left|B_{j}(u)\right|^{2} f_{n}(u) d u$; on the other hand,

$$
\begin{aligned}
\sum_{\left(l_{1}, l_{2}\right) \in R_{2}^{\prime}} 1= & \sum_{s=1}^{n-1}(N-n-s) 1_{\{N-n>n-1\}} \\
& +\sum_{s=1}^{N-n-1}(N-n-s) 1_{\{N-n \leq n-1\}} \\
\leq & |(n-1)(2 N-3 n)|+(N-n)(N-n-1) 1_{\{N-n \leq n-1\}} \\
\leq & 20 n N
\end{aligned}
$$

therefore $w_{2}=O(1 / N)$ uniformly in $\omega$ and $k$, by Lemma 7.6. In the same way $U_{n, N, 1}^{(5)}(k, \omega)=O(1 / N)$.

Now,

$$
\begin{aligned}
& \left|U_{n, N, 2}(k, \omega)\right| \\
& \leq \frac{4}{N^{2}} \sum_{\left(l_{1}, l_{2}\right) \in R^{\prime}} \sum_{j_{1}, j_{2} \in \mathbb{Z}} E\left\{\left|B_{j_{1}}\left(t_{l_{2}}-t_{l_{1}}\right) B_{j_{2}}\left(t_{l_{2}+n}-t_{l_{1}+n}\right)\right|\right\} \\
& \times\left|\phi\left(\frac{2 \pi}{T}\left(j_{1}+j_{2}\right)\right)\right|^{l_{1}}
\end{aligned}
$$




$$
\begin{aligned}
& \leq \frac{4}{N^{2}} \sum_{\left(l_{1}, l_{2}\right) \in R_{2}^{\prime}}\left(\sum_{j_{1}+j_{2} \neq 0} \frac{\left(c_{j_{1}}\left(\left|l_{2}-l_{1}\right|\right) c_{j_{2}}\left(\left|l_{2}-l_{1}\right|\right)\right)^{1 / 2}}{\left|j_{1}+j_{2}\right|}\right. \\
& \left.+\sum_{j \in \mathbb{Z}} c_{j}\left(\left|l_{2}-l_{1}\right|\right)\right) \\
& \leq \frac{8}{N^{2}} \sum_{s=0}^{N-n-1}(N-n-s)\left(\sum_{j_{1}+j_{2} \neq 0} \frac{\left(c_{j_{1}}(s) c_{j_{2}}(s)\right)^{1 / 2}}{\left|j_{1}+j_{2}\right|}+\sum_{j \in \mathbb{Z}} c_{j}(s)\right) \\
& \leq \frac{8}{N} \sum_{c=0}^{\infty}\left(\sum_{j_{1}+j_{2} \neq 0} \frac{\left(c_{j_{1}}(s) c_{j_{2}}(s)\right)^{1 / 2}}{\left|j_{1}+j_{2}\right|}+\sum_{j \in \mathbb{Z}} c_{j}(s)\right)=O\left(\frac{1}{N}\right) .
\end{aligned}
$$

Next,

$$
\begin{aligned}
U_{n, N, 3}(k, \omega) \leq & \frac{4}{N^{2}} \sum_{\left(l_{1}, l_{2}\right) \in R^{\prime}} \sum_{j_{1}+j_{2} \neq 0} \frac{\left(c_{j_{1}}\left(\left|l_{2}-l_{1}+n\right|\right) c_{j_{2}}\left(\left|l_{2}-l_{1}-n\right|\right)\right)^{1 / 2}}{\left|j_{1}+j_{2}\right|} \\
& +\frac{4}{N^{2}} \sum_{\left(l_{1}, l_{2}\right) \in R^{\prime}} \sum_{j \in \mathbb{Z}}\left(c_{j}\left(\left|l_{2}-l_{1}+n\right|\right) c_{j}\left(\left|l_{2}-l_{1}-n\right|\right)\right)^{1 / 2} \\
\leq & \frac{8}{N^{2}} \sum_{s=0}^{N-n-1}\left(\sum_{j_{1}+j_{2} \neq 0} \frac{\left(c_{j_{1}}(|s+n|) c_{j_{2}}(|s-n|)\right)^{1 / 2}}{\left|j_{1}+j_{2}\right|}+\sum_{j \in \mathbb{Z}}\left(c_{j}(|s+n|) c_{j}(|s-n|)\right)^{1 / 2}\right) \\
\leq & \frac{8}{N} \sum_{c=0}^{\infty}\left(\sum_{j_{1}+j_{2} \neq 0} \frac{\left(c_{j_{1}}(s) c_{j_{2}}(s)\right)^{1 / 2}}{\left|j_{1}+j_{2}\right|}+\sum_{j \in \mathbb{Z}} c_{j}(s)\right)=O\left(\frac{1}{N}\right) .
\end{aligned}
$$

Finally,

$$
\begin{aligned}
\left|U_{n, N, 4}(k, \omega)\right| & \leq \frac{8}{N^{2}} \sum_{\left(l_{1}, l_{2}\right) \in R^{\prime}} \sum_{j \in \mathbb{Z}} E\left\{h_{j}\left(\left|t_{l_{1}+n}-t_{l_{1}}\right|,\left|t_{l_{2}}-t_{l_{1}}\right|,\left|t_{l_{2}+n}-t_{l_{1}}\right|\right) t\right\} \\
& \leq \frac{8}{N^{2}} \sum_{\left(l_{1}, l_{2}\right) \in R^{\prime}}\left(\sum_{j \neq k} \frac{s_{j}\left(\left|l_{2}-l_{1}\right|\right)}{|j-k|}+s_{k}\left(\left|l_{2}-l_{1}\right|\right)\right)^{\times\left|\phi(j-k)^{l_{1}}\right|}
\end{aligned}
$$

where

$$
s_{j}(n)=\int_{0}^{\infty} h_{j}(0, u, 0) f_{n}(u) d u \quad \text { and } \quad s_{j}(0)=h_{j}(0,0,0) .
$$

As for $U_{n, N, 2}(k, \omega)$, we have $U_{n, N, 4}(k, \omega)=O(1 / N)$ uniformly in $\omega$ and $k$. 


\section{References}

[1] D. Dehay, Spectral analysis of the covariance kernel of the almost periodically correlated processes, Stochastic Process. Appl., submitted.

[2] H. L. Hurd, An investigation of periodically correlated processes, Ph.D. Dissertation, Duke Univ., Durham, N.C., 1969.

[3] - Periodically correlated processes with discontinuous correlation functions, Theory Probab. Appl. 19 (1974), 804-807.

[4] —, Nonparametric time series analysis for periodically correlated processes, IEEE Trans. Inform. Theory 35 (1989), 350-359.

[5] E. Masry, Poisson sampling and spectral estimation of continuous-time parameter processes, ibid. 24 (1978), 173-183.

[6] E. Masry and M. C. Lui, Discrete-time spectral estimation of continuous parameter -A new consistent estimate, ibid. 22 (1976), 298-312.

[7] F. Messaci, Estimation de la densité spectrale d'un processus en temps continu par échantillonnage poissonnien, Ph.D. Dissertation, Rouen Univ., 1986.

[8] H. S. Shapiro and R. A. Silverman, Alias-free sampling of random noise, J. Soc. Indust. Appl. Math. 8 (1960), 225-248.

VINCENT MONSAN

LABORATOIRE D'ANALYSE ET MODÈLES STOCHASTIQUES

URA CNRS 1378

UNIVERSITÉ DE ROUEN

F-76821 MONT SAINT AIGNAN CEDEX, FRANCE

Received on 21.1.1993 